\title{
Neogenin regulates neuronal survival through DAP kinase
}

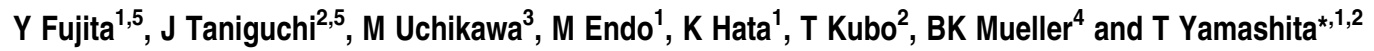

The repulsive guidance molecule (RGM) is a membrane-bound protein that has diverse functions in the developing central nervous system. Identification of neogenin as a receptor for RGM provided evidence of its cell death-inducing activity in the absence of RGM. Here, we show that the serine/threonine kinase death-associated protein kinase (DAPK) is involved in the signal transduction of neogenin. Neogenin interacts with DAPK and reduces DAPK autophosphorylation on Ser308 in vitro. Neogenininduced cell death is abolished in the presence of RGM or by blocking DAPK. Although neogenin overexpression or RGM downregulation in the chick neural tube in vivo induces apoptosis, coexpression of the dominant-negative mutant or smallinterference RNA of DAPK attenuates this proapoptotic activity. Thus, RGM/neogenin regulates cell fate by controlling the DAPK activity.

Cell Death and Differentiation (2008) 15, 1593-1608; doi:10.1038/cdd.2008.92; published online 27 June 2008

The repulsive guidance molecule (RGM) is a glycosylphosphatidylinositol -anchored glycoprotein that does not share significant homology with any other proteins. RGM was originally identified as a membrane-bound protein having repulsive and growth cone collapse-inducing activities in the chick retinotectal system. ${ }^{1}$ This molecule was later purified and cloned from the posterior part of the chick tectum. ${ }^{2}$ RGM contains a putative autoproteolytic or an unstable cleavage site, and the cleaved mature protein has a molecular mass of $33 \mathrm{kDa}$.

Recently, neogenin was identified as an RGM receptor; ${ }^{5}$ this protein was originally isolated from embryonic chicken cerebellum as a deleted in colorectal cancer (DCC) homologue. ${ }^{6}$ During central nervous system (CNS) development, neogenin mediates axon guidance by binding either RGM or netrin $-1^{7}$ and controls neuronal differentiation and survival. ${ }^{8,9}$ In the ligand-free state, neogenin induces apoptosis; ${ }^{10}$ therefore, it belongs to the structurally unrelated, functionally defined family of 'dependence receptors'. This indicates that the absence of a ligand induces self-activation of the receptor with its subsequent proteolytic processing, thereby triggering apoptotic cell death. ${ }^{11,12}$ Neogenin overexpression or RGMa downregulation at E1.5 in the area of the developing chick neural tube resulted in an increase in the number of terminal deoxynucleotidyl transferase-mediated dUTP nick end labeling (TUNEL)-positive cells. ${ }^{10}$ However, the signal transduction mechanism that induces cell death is completely unknown and remains to be analyzed.
When attempting to elucidate the function of RGM and neogenin, we identified the serine/threonine kinase deathassociated protein kinase (DAPK) to be an interactor of neogenin. DAPK is a crucial intracellular protein that mediates cell death by its serine threonine kinase activity. ${ }^{13-16}$ DAPK has a multidomain structure that comprises a kinase domain, a calmodulin (CaM)-binding motif, eight ankyrin repeats, a cytoskeleton-binding region, and a death domain; the kinase and death domains are both important for the proapoptotic activity. ${ }^{14,17}$ DAPK-mediated apoptosis is induced by a variety of stimuli such as interferon- $\gamma$, TNF- $\alpha$, TGF- $\beta$, ceramide, and the oncogenes c-myc and E2F. In this study, we provide evidence that DAPK is required for the proapoptotic activity of neogenin in vitro and in vivo.

\section{Results}

The ICD of neogenin interacts with DAPK. We first examined whether neogenin interacts with DAPK. Human embryonic kidney (HEK) 293 cells endogenously express DAPK (Figure 1a), as previously reported, ${ }^{4}$ and these cells were used for coimmunoprecipitation experiments. The fulllength neogenin was transfected into HEK293 cells. In the DAPK immunoprecipitates, an anti-neogenin antibody revealed of neogenin (Figure 1a). To test whether the interaction depends on ligand binding, we treated the cells with soluble RGMa and found that the interaction reduced

\footnotetext{
${ }^{1}$ Department of Molecular Neuroscience, Graduate School of Medicine, Osaka University, 2-2 Yamadaoka, Suita, Osaka 565-0871, Japan; ${ }^{2}$ Department of Neurobiology, Graduate School of Medicine, Chiba University, 1-8-1 Inohana, Chuo-ku, Chiba 260-8670, Japan; ${ }^{2}$ Laboratory for Developmental Biology, Graduate School of Frontier Biosciences, Osaka University, 1-3 Yamadaoka, Suita, Osaka 565-0871, Japan and ${ }^{4}$ Neuroscience Discovery, Abbott GmbH and Company KG, Ludwigshafen 67061, Germany

*Corresponding author: T Yamashita, Department of Molecular Neuroscience, Graduate School of Medicine, Osaka University, 2-2 Yamadaoka, Suita, Osaka 565-0871, Japan. Tel: + 81-6-6879-3660; Fax: 81-6-6879-3669; E-mail: yamashita@molneu.med.osaka-u.ac.jp

${ }^{5}$ These authors contributed equally to this work.

Keywords: RGM; neogenin; DAPK; neuron

Abbreviations: RGM, repulsive guidance molecule; CNS, central nervous system; DAPK, death-associated protein kinase; DCC, deleted in colorectal cancer; siRNA, small-interference RNA; TUNEL, terminal deoxynucleotidyl transferase-mediated dUTP nick end labeling; CaM, calmodulin; ICD, intracellular domain; HEK, human embryonic kidney; CGN, cerebellar granule neuron; HH, Hamburger Hamilton; MLC, myosin light chain; GFP, green fluorescent protein; DMEM, Dulbecco's modified Eagle's medium; FBS, fetal bovine serum; PFA, paraformaldehyde; BSA, bovine serum albumin; DAPI, 4',6'-diamidino-2-phenylindole

Received 30.11.07; revised 28.4.08; accepted 29.5.08; Edited by L Greene; published online 27.6.08
} 


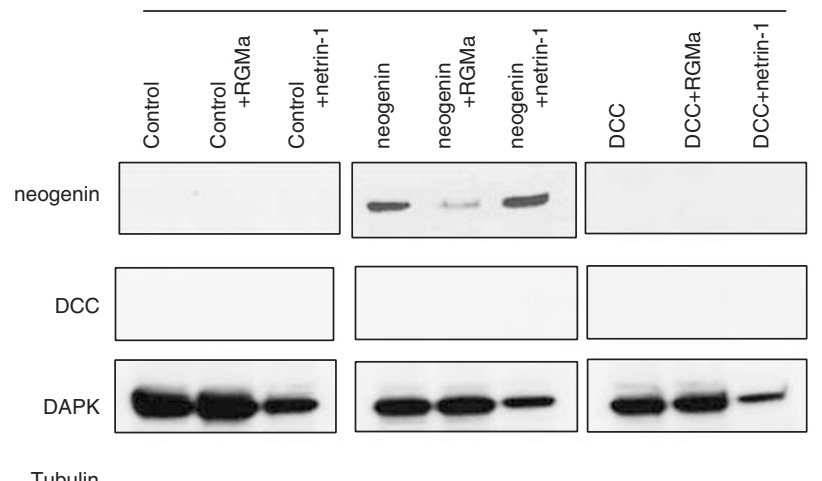

Total lysates

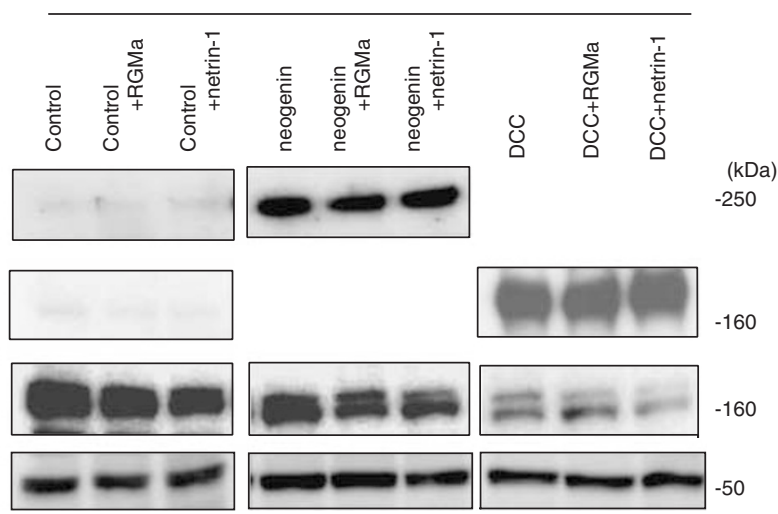

b

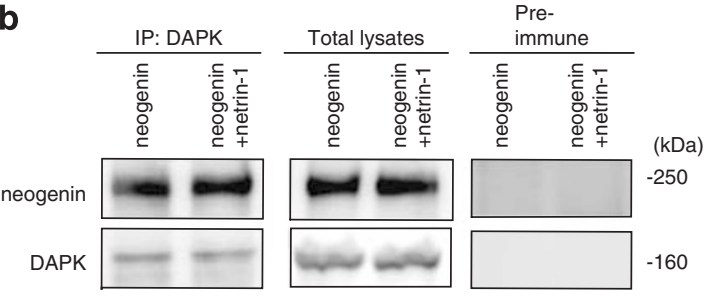

d
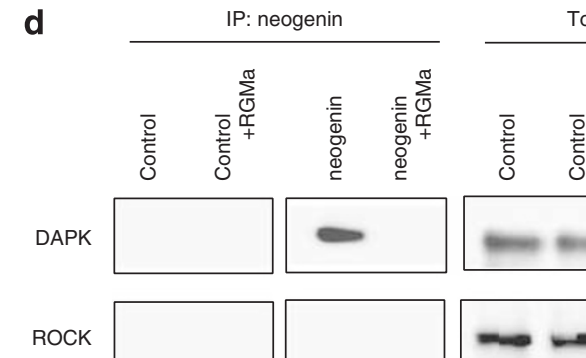

Total lysates
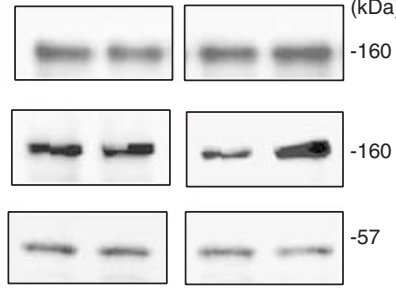

JNK
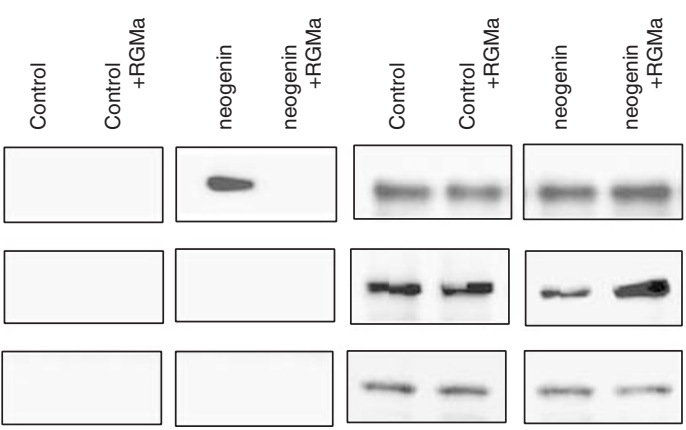

$\stackrel{8}{\square}$
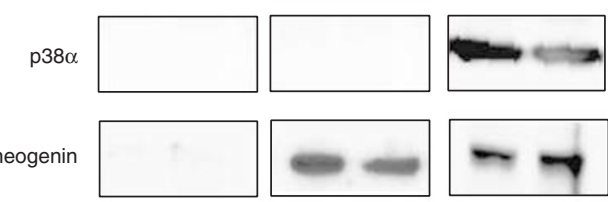

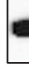

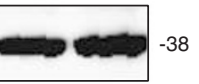
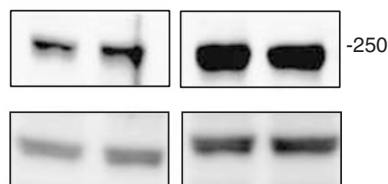

Tubulin

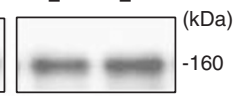

e
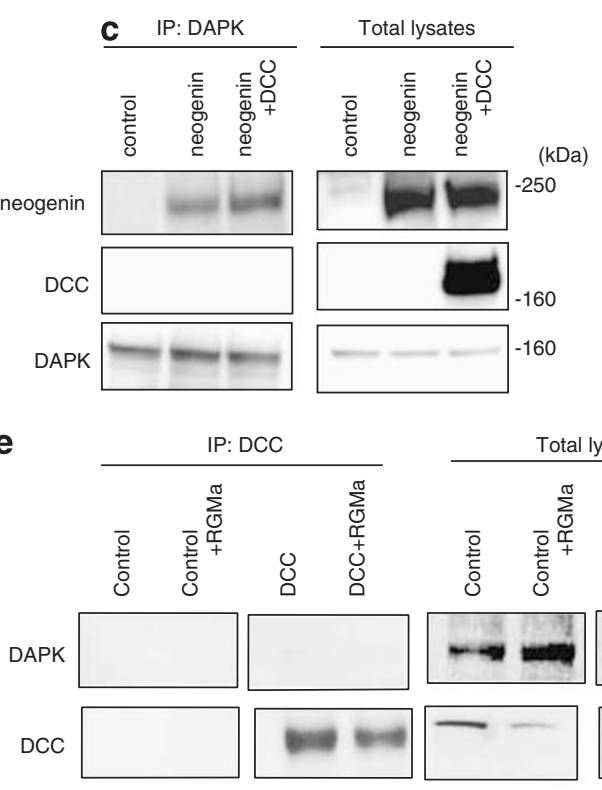

Total lysates
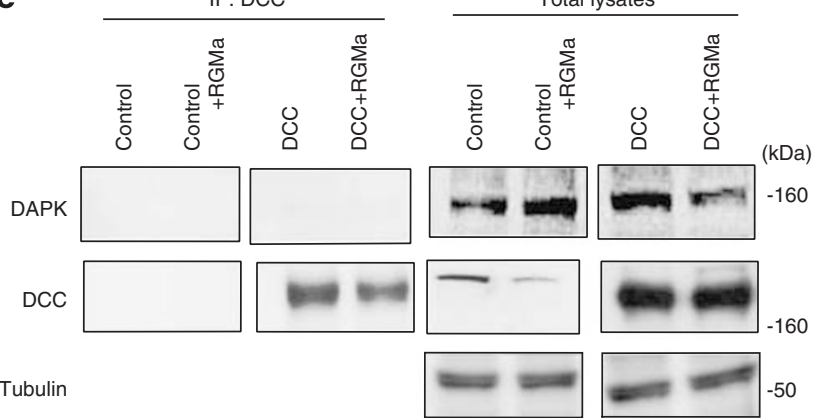

Figure 1 Neogenin interacts with death-associated protein kinase (DAPK) in human embryonic kidney (HEK) 293 cells. (a) HEK293 cells were transiently transfected with an empty vector (control), VSV-G-tagged neogenin, or deleted in colorectal cancer (DCC). At $48 \mathrm{~h}$ after the transfection, the cells were treated with or without soluble RGMa $(1 \mu \mathrm{g} / \mathrm{ml})$ or soluble netrin-1 $(1 \mu \mathrm{g} / \mathrm{ml})$ for $30 \mathrm{~min}$. Immunoprecipitation of DAPK followed by western blot with the anti-neogenin antibody (upper panel), the anti-DCC antibody (the second panel), or the anti-DAPK antibody (the third panel). Representative pictures from three independent experiments show that neogenin, but not DCC, was coimmunoprecipitated with DAPK. Treatment of the cells with RGMa $(1 \mu \mathrm{g} / \mathrm{ml})$ decreased the binding, whereas netrin- $1(1 \mu \mathrm{g} / \mathrm{ml})$ had no effect. The bottom panels show tubulin staining. The panels on the right side represent expression of the corresponding proteins in the lysates. (b) The neogenin/DAPK interaction was not changed by netrin1. (c) The neogenin/DAPK interaction was not changed if DCC was present. (d) Immunoprecipitation of neogenin followed by western blot with the anti-DAPK antibody, the anti-Rho-kinase (ROCK) antibody, the anti-JNK antibody, the anti-p38 $\alpha$ antibody, or the anti-neogenin antibody (neogenin). Representative pictures from three independent experiments show that association of DAPK with neogenin was observed in the absence of RGMa, whereas the binding was decreased by the RGMa treatment (1 $\mu \mathrm{g} / \mathrm{ml})$. The panels on the right side represent expression of the corresponding proteins in the lysates. (e) Immunoprecipitation of DCC followed by western blot with the anti-DAPK antibody. No signal for DAPK was observed

due to RGMa treatment. However, treatment with soluble netrin-1 did not reduce the interaction (Figures $1 \mathrm{a}$ and $\mathrm{b}$ ). Further, the neogenin/DAPK interaction was not modified if DCC was present (Figure 1c). DAPK was coimmunoprecipitated with neogenin when the antineogenin antibody was used for pull down (Figure 1d). Reduced association was observed in the RGMa-treated cells, as in the case mentioned above. Other kinases 
including Rho-kinase, JNK, or p38 were not coimmunoprecipitated with neogenin (Figure 1d). In addition, we observed no signal for the interaction of DAPK and DCC (Figures 1a and e). These results demonstrate that ectopically expressed neogenin constitutively associates with endogenous DAPK and that RGM binding to neogenin abolishes this interaction.

We then examined the interaction of these molecules by using lysates prepared from cerebellar granule neurons (CGNs), which express both proteins endogenously, from postnatal day (P) 7-8. DAPK was coimmunoprecipitated with neogenin, but was not coimmunoprecipitated when the cells were treated with RGMa or when DCC was immunoprecipitated (Figure 2a). Neogenin was coimunoprecipitated with DAPK if DAPK was immunoprecipitated with the antibody against DAPK (Figure 2a). Next, we decided to perform immunodepletion of DAPK and neogenin to examine interaction of these molecules in CGNs. If DAPK binds to neogenin constitutively, the amount of free DAPK in the lysates should be reduced by the neogenin immunodepletion. Immunodepletion was performed using an antibody against neogenin, followed by western blot for DAPK (Figure $2 b$; left). The result shows that the amount of DAPK in the supernatant was reduced after immunodepletion of neogenin, whereas the prior RGMa treatment significantly increased it. The amount of neogenin decreased after immunodepletion of DAPK
(Figure 2b; right). These data demonstrate that neogenin constitutively interacts with DAPK in rat CGNs. Moreover, RGMa appears to regulate the neogenin/DAPK interaction.

We further assessed interaction of these molecules by using lysates prepared from the mesencephalon from Hamburger Hamilton (HH) stage 17 chick embryos. Consistent with the observations in the CGNs, neogenin was coimmunoprecipitated with DAPK (Figure 2c).

To assess direct interaction of these two molecules, we employed the yeast two-hybrid system. The yeast reporter strain $\mathrm{AH} 109$ was cotransformed with a bait comprising the full intracellular domain (ICD) of neogenin fused to the GAL4 DNA-BD (pGBKT7-neogenin ICD) and a prey comprising DAPK fused to the GAL4 AD (pGADT7-DAPK). Strong growth of the yeast was detected only when both constructs were transformed (data not shown), demonstrating that neogenin ICD interacts with DAPK. Then, several deletion mutants of DAPK fused to the GAL4 AD (pGADT7-DAPK 1-275, 276637, 638-847, 848-1129, 989-1270, and 1271-1423) were used to define the domains of DAPK that are necessary for the interaction. No growth was observed on adenine, histidine, tryptophan, and leucine-deficient medium in transformants of the neogenin ICD bait with DAPK 1-275, 276-637, 638-847, or 848-1129, whereas strong growth was detected with DAPK 989-1270 and 1271-1423 (Figure 3a). DAPK 1271-1423 corresponds to a so-called death domain. Thus, the death

a rat CGNs

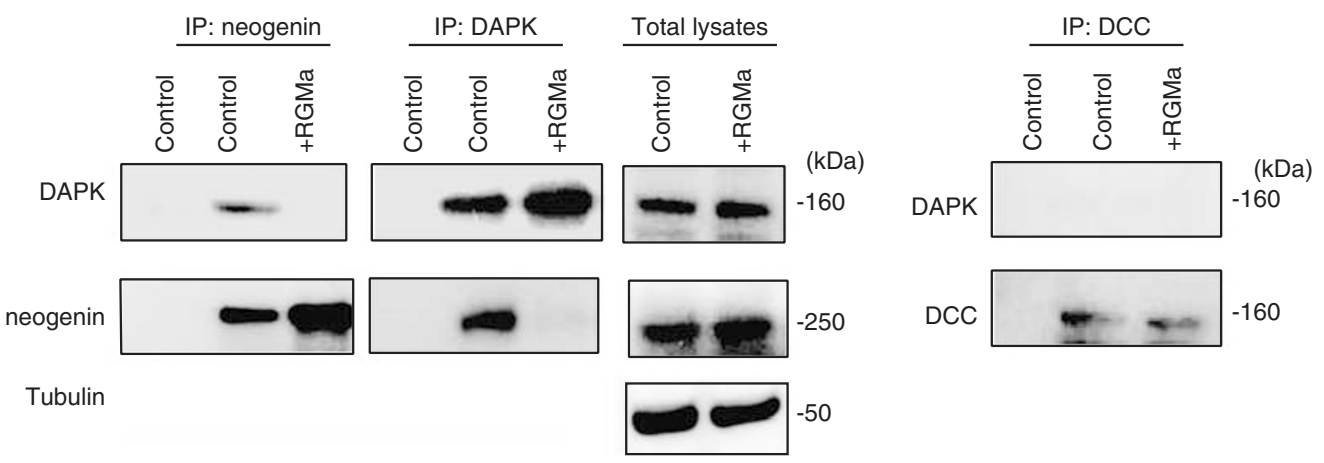

b rat CGNs

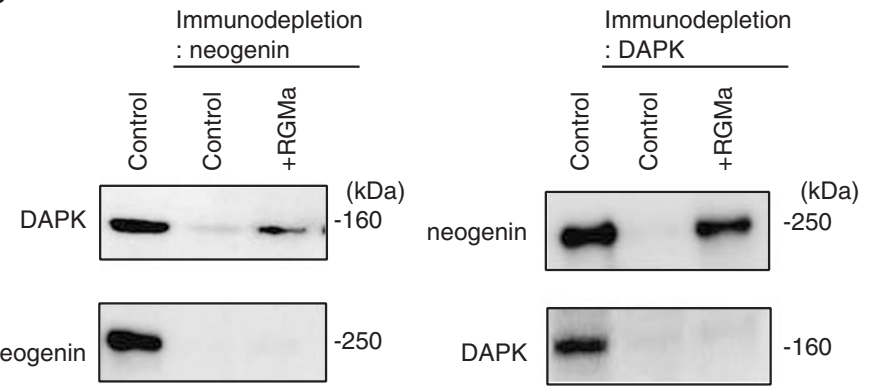

C chick brain extracts

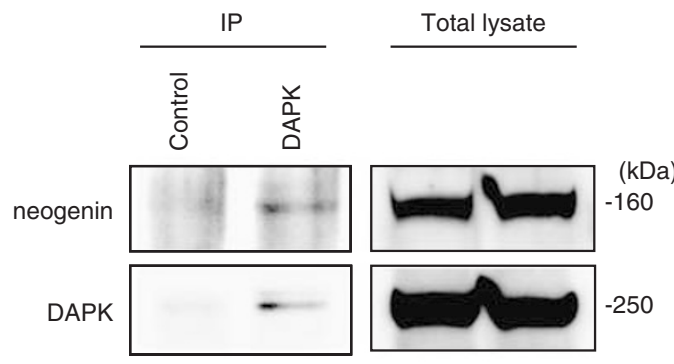

Figure 2 Neogenin interacts with death-associated protein kinase (DAPK) in rat cerebellar granule neurons (CGNs) and chick brain. (a) Lysates from rat CGNs were immunoprecipitated with control IgG (control), anti-neogenin (IP: neogenin), anti-DAPK (IP: DAPK), or anti-deleted in colorectal cancer (DCC) antibody (IP: DCC). Representative pictures from three independent experiments are shown. DAPK was coimmunoprecipitated with neogenin, but was not coimmunoprecipitated when the cells were treated with RGMa $(1 \mu \mathrm{g} / \mathrm{ml})$. DAPK was not coimmunoprecipitated with DCC. (b) Lysates from rat CGNs were immunodepleted with the anti-neogenin (neogenin; left) or the anti-DAPK (DAPK; right). Representative pictures from three independent experiments are shown. The amount of DAPK in the supernatant (upper) was reduced after immunodepletion of neogenin (lower), whereas that the RGMa $(1 \mu \mathrm{g} / \mathrm{ml})$ treatment significantly increased it. Consistent with this observation, the amount of neogenin decreased when DAPK was immunodepleted. (c) Coimmunoprecipitation of neogenin with DAPK from lysates prepared from the mesencephalon from Hamburger Hamilton $(\mathrm{HH})$ stage 17 chick embryos. Immunoprecipitation was performed using an antibody against DAPK, followed by western blot for neogenin 
domain or DAPK $989-1270$ is sufficient for the interaction with neogenin.

We then tried to define the domains of neogenin that are necessary for the interaction with the death domain of DAPK. The ICD of neogenin has P1, P2, and P3 domains that are functionally important. ${ }^{18}$ Various deletion constructs of neogenin ICD were used as shown in Figure $3 \mathrm{~b}$. The results demonstrate that both $\mathrm{P} 2$ and $\mathrm{P} 3$ domains were necessary for the interaction with the death domain of DAPK (Figure 3b).

We further assessed direct interaction of DAPK with neogenin ICD using recombinant proteins. Three deletion mutants of His-tagged DAPK (848-1129, 989-1270, and 12711423; Figure 3c left panel) and GST-fused neogenin ICD were purified and used for pull-down assays. The death domain of DAPK was coprecipitated with GST-fused ICD of neogenin
(Figure 3c). However, we could not detect interaction of neogenin ICD with DAPK 989-1270 or DAPK 848-1129 (Figure 3c).

RGM and neogenin regulate the activity of DAPK. DAPK is autophosphorylated on Ser308, which is located in the CaM-binding domain; this process disables the kinase activity. ${ }^{17,19}$ After demonstrating that neogenin interacts with DAPK, we proceeded to investigate the effects of neogenin on DAPK phosphorylation. To analyze whether DAPK phosphorylation is regulated by neogenin, HEK293 cells were transiently cotransfected with a control or a VSVG-tagged neogenin construct together with an HA-tagged wild-type DAPK construct. Autophosphorylation of DAPK was examined in the presence or absence of soluble RGMa by using immunodetection on western blots with a a

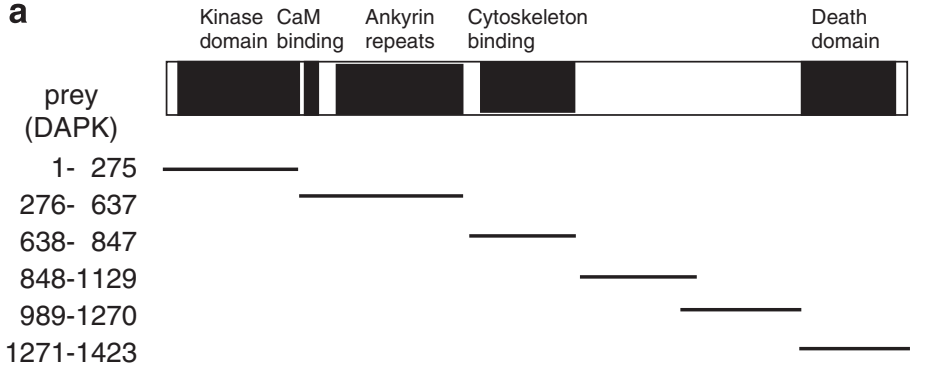

b Bait

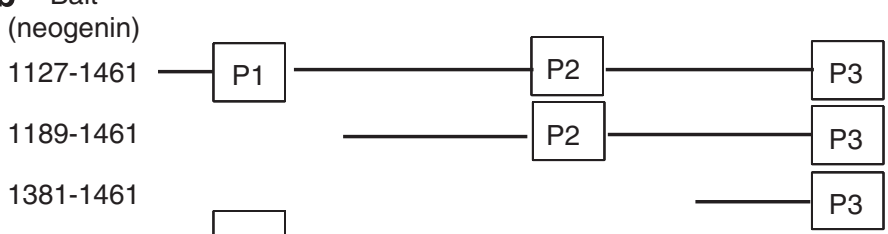

Bait SD/-Ade/-His/

-Leu/-Trp

Neo ICD -

Neo ICD

Neo ICD

Neo ICD

Neo ICD

Neo ICD

Prey SD/-Ade/-His/ (DAPK) -Leu/-Trp

1271-1423 +

1271-1423 +

$1271-1423$

1271-1423

1271-1423

1271-1423
$-$

$+$

$+$

1127-1407

$1127-1449$<smiles>c1ccccc1</smiles>

P1

P2

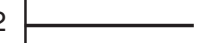

C
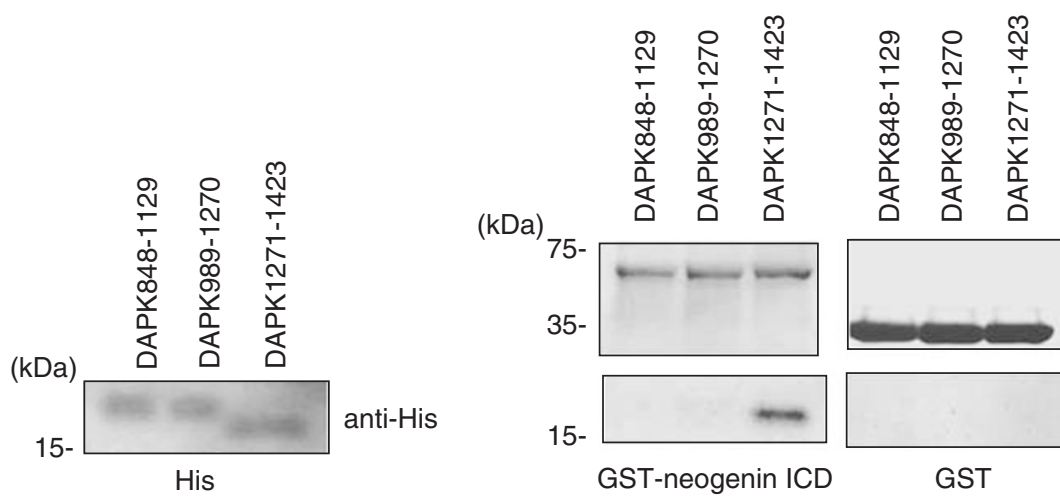

anti-GST

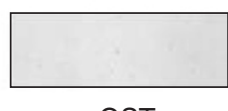

anti-His

Figure 3 Direct interaction of death-associated protein kinase (DAPK) and neogenin. (a) Schematic representation of DAPK deletion constructs and the neogenin intracellular domain (ICD) for the yeast two-hybrid assay. Strong growth was observed in transformants of the neogenin ICD and DAPK (989-1270 or 1271-1423). Excellent ability to grow on adenine (Ade) is denoted by + , histidine (His), leucine (Leu), and tryptophan (Trp)-deficient medium, and -, no growth. (b) Schematic representation of neogenin cytoplasmic deletion constructs and the DAPK death domain (1271-1423) for the yeast two-hybrid assay. Strong growth was observed in transformants of the death domain of DAPK (1271-1423) and neogenin ICD (1127-1461 or 1189-1461). (c) In vitro binding assay for neogenin and DAPK. The purified His-tagged DAPK proteins (8481129, 989-1270, 1271-1423; left panel) were incubated with either GST or GST-neogenin ICD. GST-fused proteins were precipitated, and the pull-down products were analyzed by western blots (right panels). Immunoblot for His-tagged proteins with the anti-His antibody demonstrates that DAPK 1271-1423 binds to the neogenin ICD, whereas DAPK 848-1129 and DAPK 989-1270 do not (right) 
monoclonal antibody for specifically detecting phosphorylation of the DAPK Ser308 residue. Neogenin expression in HEK293 cells resulted in the inhibition of DAPK autophosphorylation on Ser308 (Figure 4a), whereas the presence of RGMa completely restored DAPK autophosphorylation (Figure 4a). Next, we performed kinase assays to assess the activity of DAPK using myosin light chain (MLC) as a substrate. The HEK293 cells were transfected with wild-type DAPK and VSV-G-tagged neogenin, and were treated with soluble RGMa. Transfected DAPK was immunoprecipitated from the cell lysates, and the phosphorylation of MLC by the immunoprecipitated DAPK was measured. Overexpression of neogenin resulted in enhancement of the activity of DAPK, whereas the RGMa treatment reduced the activity (Figure $4 \mathrm{~b}$ ). We further assessed this issue by employing the CGNs that express neogenin and DAPK endogenously. Autophosphorylation of DAPK was examined in the presence or absence of soluble RGMa. The presence of RGMa enhanced DAPK autophosphorylation (Figure 4c). Thus, the ligand-receptor pair RGMa/neogenin regulates autophosphorylation and the activation of DAPK.

Neogenin proapoptotic activity is mediated by DAPK in HEK293 cells. The above-mentioned results suggest importance of DAPK in neogenin-mediated cell death. We further examined whether full-length neogenin displays a proapoptotic activity in HEK293 cells. To address this, TUNEL staining was performed $48 \mathrm{~h}$ after transfection in combination with staining with an anti-VSV-G antibody and $4^{\prime}, 6^{\prime}$-diamidino-2-phenylindole (DAPI). The number of TUNEL-positive cells was significantly increased in HEK293 cells that were transfected with the full-length neogenin construct, whereas the addition of RGMa inhibited the proapoptotic effect of neogenin (Figures 5a-e). Approximately $40 \%$ of the cells were TUNEL positive by the neogenin overexpression (Figure $5 d$ ). We subsequently analyzed whether the inhibition of endogenous DAPK activity possibly modulates neogenin-mediated cell death. A kinasedead mutant (K42A) was used as a dominant-negative form a
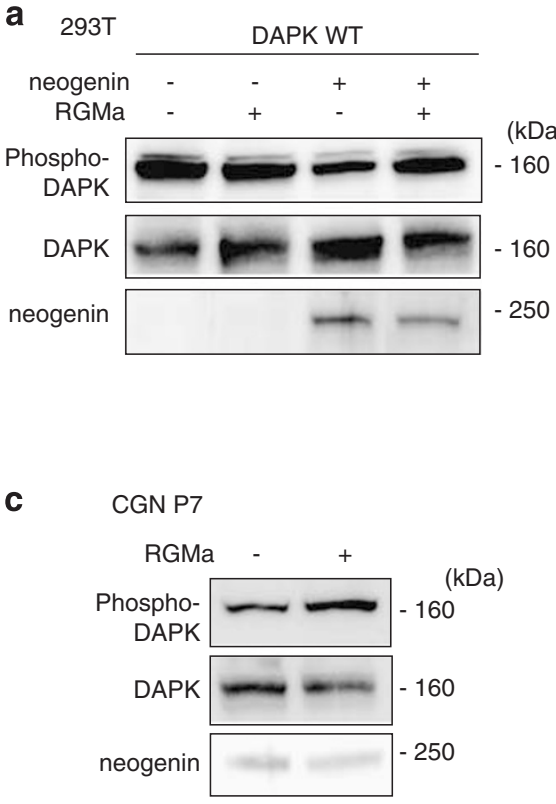

b
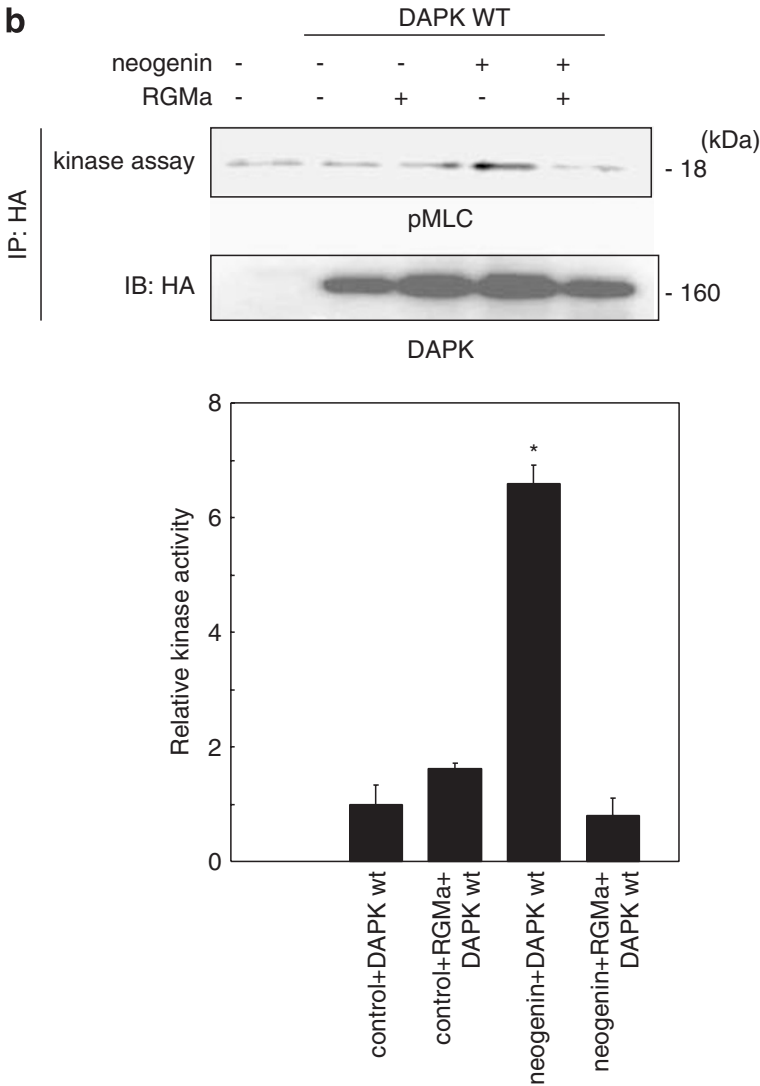

Figure 4 Repulsive guidance molecule (RGM) and neogenin regulate the activity of death-associated protein kinase (DAPK). (a) DAPK phosphorylation is regulated by neogenin and RGMa. Human embryonic kidney (HEK) 293 cells were cotransfected with the empty vector (-) or VSV-G-tagged neogenin together with HA-tagged DAPK (DAPK wild type (wt)), and were treated with or without RGMa $(1 \mu \mathrm{g} / \mathrm{ml})$. Western blotting was performed using the anti-DAPK antibody (DAPK) and the anti-phospho-DAPK antibody (phospho-DAPK). Ectopic expression of neogenin decreased the DAPK autophosphorylation, whereas treatment of RGMa restored this autophosphorylation. (b) Quantification of the DAPK activity. The HA-tagged DAPK was immunoprecipitated from cell lysates, and subjected to in vitro kinase reaction using myosin light chain (MLC) as the substrate. The MLC phosphorylation was detected by western blot (upper panel). The same kinase reactions were subjected to western blot to detect the precipitated HAtagged DAPK (lower panel). The band intensities for MLC phosphorylation were measured, and the background intensity corresponding to MLC phosphorylation without DAPK was subtracted. The relative kinase activity is shown in the graph. Results are represented as mean \pm S.E.M. $(n=3) .{ }^{*} P<0.05$; one-way ANOVA followed by Scheffe's multiple comparison test. DAPK wt: HA-tagged DAPK. (c) The cerebellar granule neuron (CGNs) were treated with or without RGMa (1 $\mu \mathrm{g} / \mathrm{ml})$. Western blotting was performed using the anti-DAPK antibody (DAPK) and the anti-phospho-DAPK antibody (phospho-DAPK). The treatment of RGMa enhanced the DAPK autophosphorylation 
a

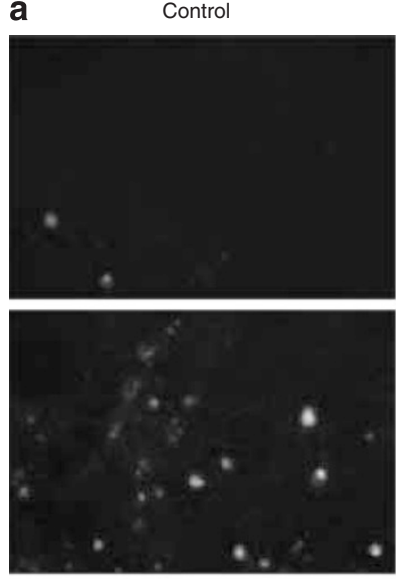

neogenin

b
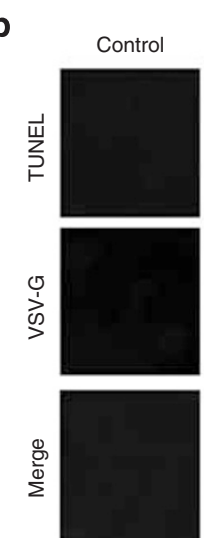

Control

RGMa
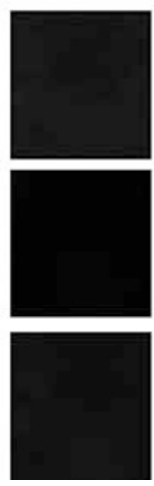

c
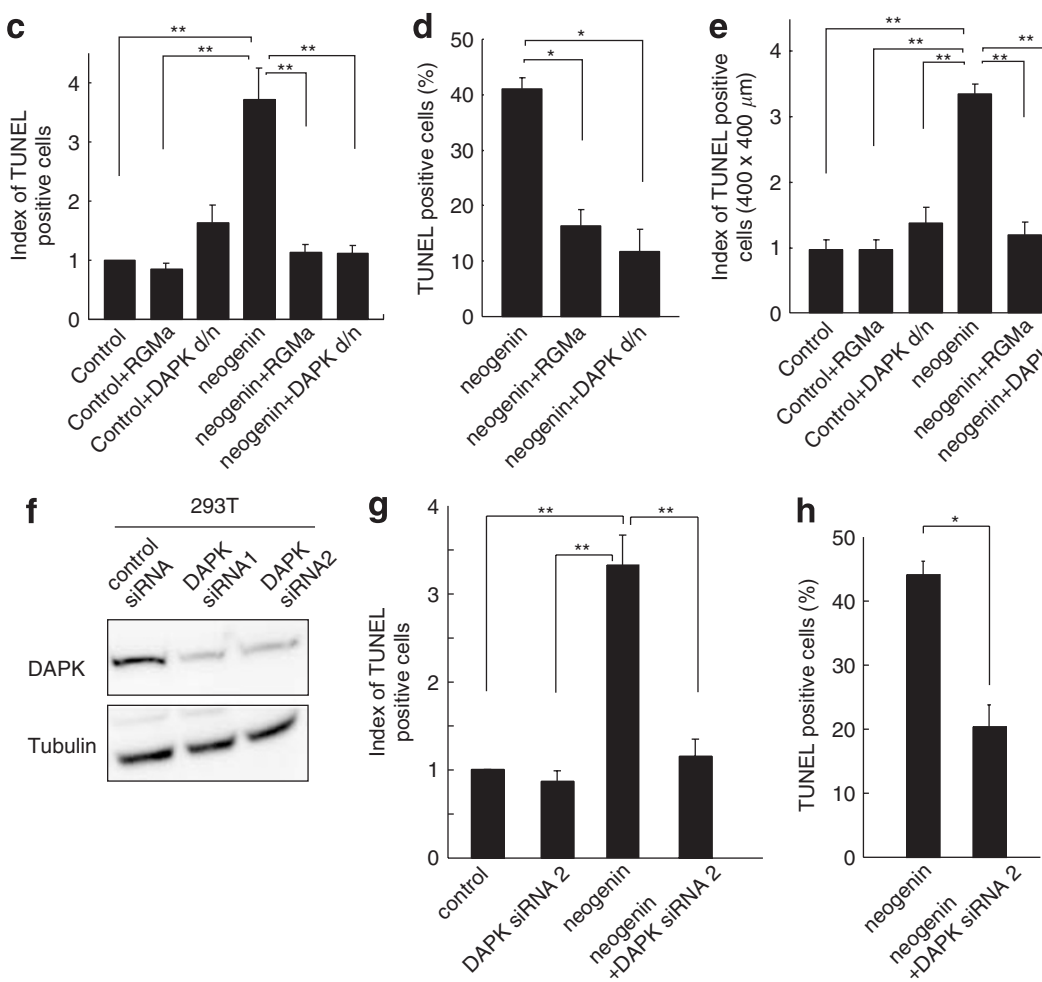
of DAPK (DAPK-d/n). ${ }^{20}$ The neogenin-mediated increase in the number of TUNEL-positive cells was completely inhibited in the presence of this dominant-negative mutant (Figures $5 a-e)$, whereas the expression level of neogenin was comparable (Figure $5 \mathrm{~b}$ ). By itself, DAPK-d/n did not have any effect on the number of TUNEL-positive cells (Figures $5 \mathrm{a}-\mathrm{c}$ and $\mathrm{e})$. We further assessed involvement of DAPK using the RNA interference technique. The HEK293 cells or rat CGNs were transfected with DAPK small-interference RNA (siRNA) or the control siRNA, and the expression of DAPK was determined by western blot analysis (Figures $5 f$ and $6 \mathrm{a}$ ). The result demonstrated that DAPK expression was decreased specifically after the DAPK siRNA transfection in HEK293 cells as well as rat CGNs. The neogenin-mediated increase in the number of TUNEL-positive cells was completely inhibited when DAPK siRNA was transfected (Figures $5 \mathrm{~g}$ and $\mathrm{h}$, Supplementary Figure 1), These results demonstrate that neogenin proapoptotic activity is mediated by DAPK in HEK293 cells.

To ask if absence of RGMa is associated with an increase in death of the CGNs, the CGNs were stained for TUNEL in the presence or absence of RGMa (Figure 6b). The cell death was not induced in the absence of RGMa. Knockdown of DAPK by siRNA (Figure 6a) did not influence the number of TUNELpositive cells (Figures $6 b$ and $c$ ). These results suggest that cell death induction by neogenin is cell-type specific, or that neogenin-expression level was not high enough to induce cell death. We further assessed the importance of DAPK in the CGNs. Although addition of RGMa induces growth cone collapse of the CGNs, knockdown of DAPK by siRNA did not modulate the effect of RGMa (Figures $6 \mathrm{~d}$ and e). Therefore, DAPK appears not to be involved in the RGM-mediated growth cone collapse.

Cytochrome $c$ release from the mitochondria induced by neogenin is dependent on DAPK. The release of cytochrome $c$ into the cytosol is an early step in apoptotic cell death. ${ }^{21}$ In this regard, we examined whether neogenin could induce the release of cytochrome $c$ in HEK293 cells. Immunofluorescence staining of HEK293 cells with an anticytochrome $c$ antibody yielded a punctate pattern, which is characteristic of mitochondrial localization (Figure 7a; control). However, diffuse cytochrome $c$ staining was observed in the case of cells transfected with the full-length neogenin construct (Figure 7a; neogenin). As expected, the punctate pattern of cytochrome $c$ staining was restored in the presence of RGMa or DAPK-d/n in the neogenin-expressing cells (Figure 7a). Quantification of the cells with diffuse cytochrome $c$ staining was performed. Figure $7 \mathrm{~b}$ shows the percentage of cells with diffuse cytochrome $c$ staining, and Figure 7c shows the percentage of cells with diffuse cytochrome $c$ staining among the neogenin-expressing cells. Less than $20 \%$ of the cells showed diffuse cytochrome $c$ staining among the neogenin-expressing cells (Figure 7c). As approximately $40 \%$ of the cells were TUNEL positive by the neogenin overexpression (Figure $5 \mathrm{~d}$ ), cytochrome $c$ staining might be less sensitive assay as another method for measuring cell death. Quantification of the cells with diffuse cytochrome $c$ staining among the cells not expressing neogenin following transfection of neogenin shows no induction of cell death $(0.31 \%)$ beyond the control state, suggesting that the cell death induced by neogenin is cell autonomous. We obtained consistent results when DAPK siRNA was used to block the DAPK function (Figures 7d and e and Supplementary Figure 2). We assessed this issue further using cell fractionation. Cytosolic and mitochondrial proteins were isolated, and quantified by western blot. The neogenin expression increased the cytosolic cytochrome $c$, whereas transfection of DAPK- $d / n$ or DAPK siRNA as well as the RGMa treatment attenuated this effect (Figure 7f). These results indicate that neogenin induced the release of cytochrome $c$, whereas the addition of RGMa or cotransfection of the cells with DAPK-d/n or DAPK siRNA suppressed the release.

Cell death induced by neogenin is dependent on DAPK in the chick neural tube. To test whether DAPK is involved in neogenin-mediated cell death in vivo, we analyzed cell death in neural tubes of chick embryos. We performed immunohistochemistry to investigate the distribution of DAPK in the chick neural tube. Cryostat sections at the level of the mesencephalon from $\mathrm{HH}$ stage 17 embryos were used for immunostaining. A strong immunoreactivity for RGM as well as DAPK was found in the neural tube (Figures $8 a$ and b). These immunohistochemical observations were confirmed by western blotting using lysates from the neural tube tissues (Figure 8c). We electroporated a green fluorescent protein (GFP) expression construct into a chick neural tube $(\mathrm{HH}$ stage 10). The electroporated area, which expressed GFP, covered the dorsal metencephalon, mesencephalon, and caudal diencephalons (Figure 8d). At $24 \mathrm{~h}$ after the electroporation, chick embryos were fixed and TUNEL staining was subsequently performed (Figure 8e). No significant increase in the number of TUNEL-positive cells

Figure 5 Cell death induced by neogenin is dependent on death-associated protein kinase (DAPK). (a) Terminal deoxynucleotidyl transferase-mediated dUTP nick end labeling (TUNEL) staining of the transfected human embryonic kidney (HEK) 293 cells. The cells were transfected with the empty vector (control) or VSV-G-tagged full-length neogenin constructs together with or without a dominant-negative form of DAPK (DAPK-d/n). Where indicated, RGMa $(1 \mu \mathrm{g} / \mathrm{ml})$ was added to the culture. TUNEL staining (green) was performed $48 \mathrm{~h}$ after the transfection. Scale bar: $100 \mu \mathrm{m}$. (b) TUNEL staining and presence of the transfected VSV-G-tagged full-length neogenin in HEK293 cells. VSV-G, VSV-G-tagged neogenin (red) immunostained with the anti-VSV-G antibody. Scale bar: $10 \mu \mathrm{m}$. (c, g) Neogenin-induced cell death is dependent on the DAPK activity. Overexpression of neogenin increased the number of the TUNEL-positive cells, whereas the RGMa treatment or cotransfection of DAPK-d/n or DAPK small-interference RNA (siRNA) resulted in return to the control number. The index factor is defined as the ratio of total number of TUNEL-positive cells compared with the control. Results are represented as mean \pm S.E.M. $(n=3-9)$. (d, h) The percentage of the number of TUNEL-positive cells among the cells expressing VSV-G-tagged neogenin. (e) The index factor is defined as the ratio of number of TUNEL-positive cells in the fixed square (measurement area, $400-400 \mu \mathrm{m}$ ) compared with the control. Results are represented as mean \pm S.E.M. $(n=3-9)$. ${ }^{*} P<0.05$; ${ }^{* *} P<0.01$; one-way ANOVA followed by Scheffe's multiple comparison test. (f) The HEK293 cells were transfected with DAPK siRNA or the control siRNA, and the expression of DAPK was determined by western blot 


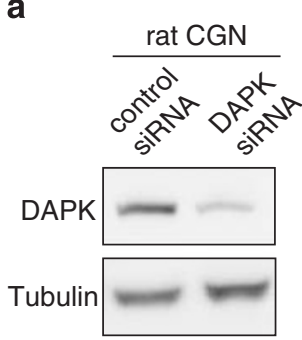

b
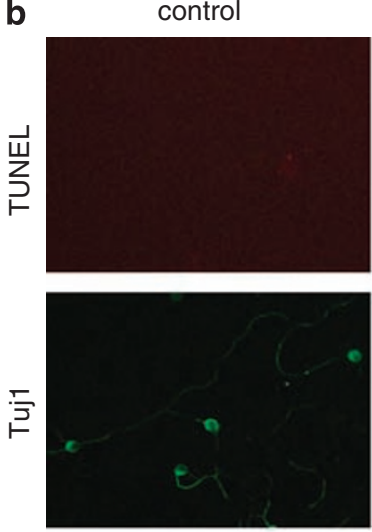
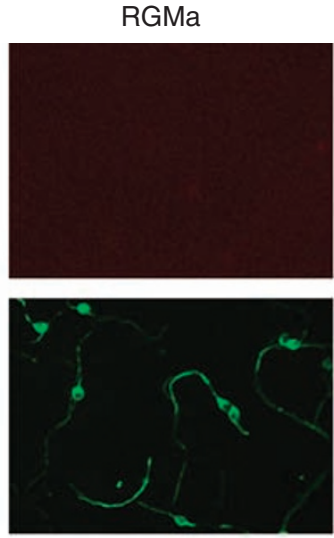

DAPK SIRNA
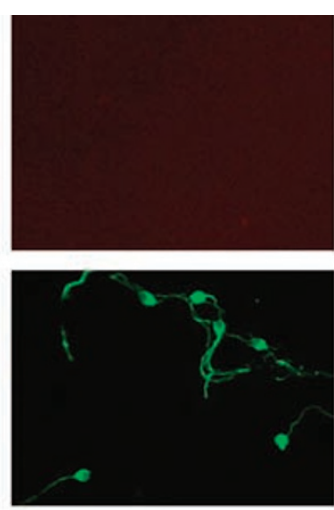

DAPK siRNA+RGMa
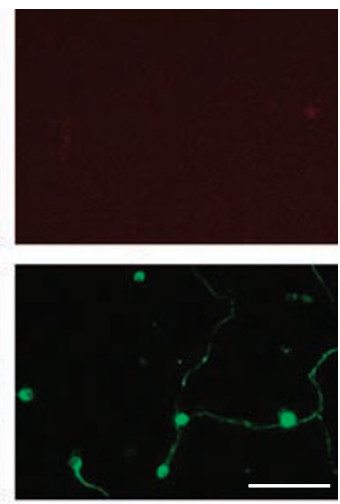

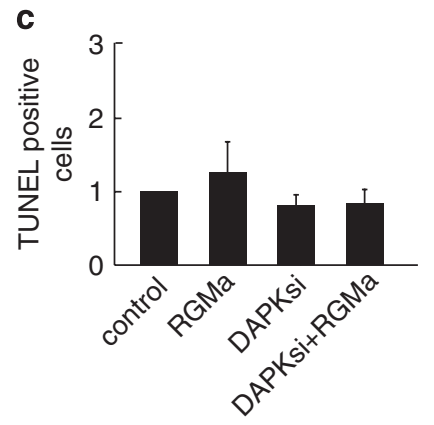

d

control

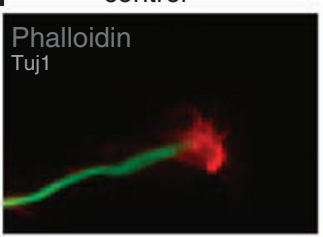

control +RGMa

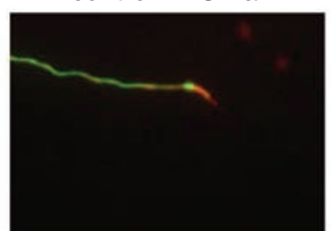

DAPK SiRNA

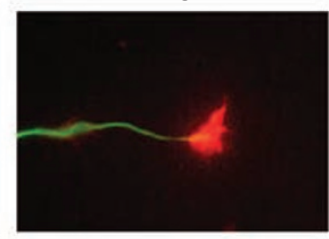

DAPK siRNA +RGMa

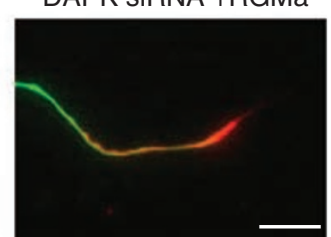

e

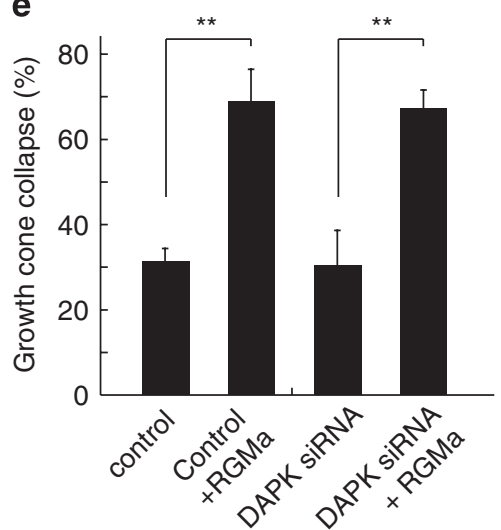

Figure 6 Death-associated protein kinase (DAPK) is not associated with cell death or growth cone collapse induced by repulsive guidance molecule (RGM) in the cerebellar granule neurons (CGNs). (a) CGNs were transfected with DAPK small-interference RNA (siRNA) or the control siRNA, and the expression of DAPK was determined by western blot. (b) Terminal deoxynucleotidyl transferase-mediated dUTP nick end labeling (TUNEL) staining of the transfected CGNs. Rat CGNs, transfected with or without DAPK siRNA, were treated with or without RGMa $(1 \mu \mathrm{g} / \mathrm{ml})$. The cells were fixed $72 \mathrm{~h}$ after the treatment, and stained for TUNEL (red) and Tuj 1 (green). No significant signal for TUNEL was observed in any conditions tested. Scale bar: $100 \mu \mathrm{m}$. (c) The percentage of the number of TUNEL-positive cells. (d) Effects of DAPK siRNA on RGMainduced growth cone collapse. The CGNs were cotransfected with DAPK siRNA or control siRNA. AT $48 \mathrm{~h}$ after the transfection, neurons were treated with RGMa ( $2 \mu \mathrm{g} / \mathrm{ml})$ or the control medium for $30 \mathrm{~min}$, fixed, and labeled with phalloidin. (e) Growth cone collapse induced by RGM was not reduced in the CGNs transfected with DAPK siRNA. ${ }^{* *} P<0.01$; one-way ANOVA followed by Scheffe's multiple comparison test

was observed in the electroporated side, which expressed GFP (right side), $24 \mathrm{~h}$ after GFP electroporation (Figure 8e).

Using this in vivo system, we then assessed whether the proapoptotic activity of neogenin in vivo is dependent on the DAPK activity. We electroporated the control or VSV-Gtagged neogenin constructs in combination with or without DAPK- $d / n$ together with the GFP-expression construct in $\mathrm{HH}$ stage 10 chick embryos. The signals for GFP (green), DAPK$\mathrm{d} / \mathrm{n}$ (red), and neogenin (blue) were observed in the electroporated side after coelectroporation of the constructs (Figures $9 \mathrm{a}$ and b). TUNEL staining was performed $24 \mathrm{~h}$ after electroporation. There was an increase in the number of TUNEL-positive cells on the experimental side after neogenin electroporation (Figures $9 c-e$ ). Coelectroporation of DAPK-d/n and VSV-G-tagged neogenin resulted in the disappearance of the TUNEL-positive cells. We obtained consistent results when DAP siRNA was electroporated (Figure 9e and Supplementary Figure 3). We then electroporated the control or VSV-G-tagged neogenin deletion constructs (neogenin ICD, neogenin ICD (1127-1323), and neogenin ICD
(1324-1461)) in combination with or without DAPK-d/n. Our yeast two-hybrid data show that P2 and P3 domains of neogenin were necessary for binding with the death domain of DAPK. Although neogenin ICD (1127-1323) contains only P1 domain, neogenin ICD (1324-1461) contains both P2 and P3 domains. As electroporation of either construct did not increase the number of the TUNEL-positive cells (Figure 9f), the $\mathrm{N}$-terminal domain of neogenin ICD may also be necessary for inducing cell death.

There was an increase in the number of TUNEL-positive cells on the experimental side after wild-type DAPK electroporation (Figures $9 \mathrm{~g}$ and $\mathrm{h}$ ). When neogenin and DAPK-d/n were simultaneously coelectroporated, there was no reduction in the expression of VSV-G-tagged neogenin as compared to VSV-G-tagged neogenin single electroporation (Figures $9 \mathrm{i}$ and $\mathrm{j}$ ). This excludes the possibility that reduction of neogenin-induced apoptosis was due to the reduced expression of neogenin. In addition, DAPK-d/n or DAPK siRNA did not influence the number of the TUNEL-positive cells by itself (Figures $9 c-e$ ). These data demonstrate that the 


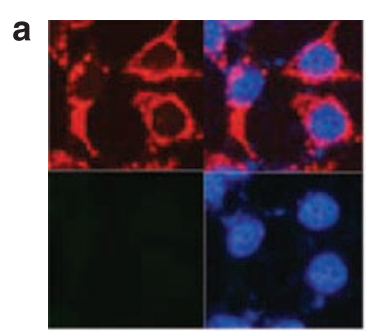

Control

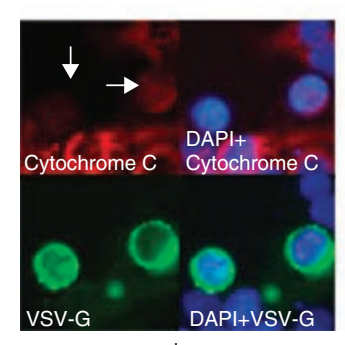

neogenin

b

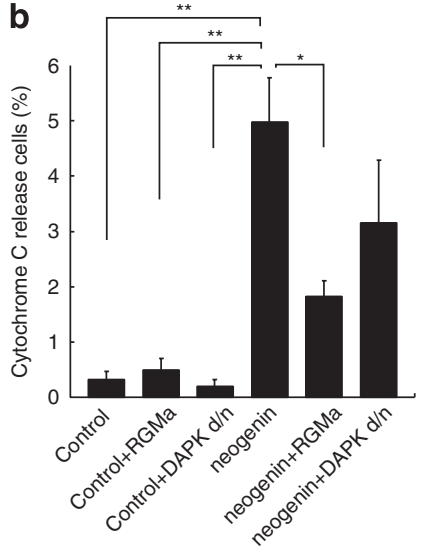

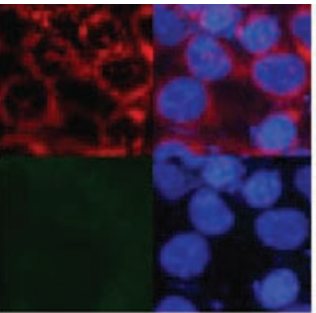

Control+RGMa

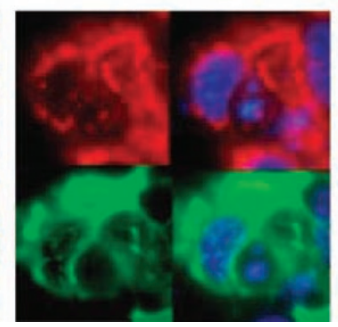

neogenin+RGMa

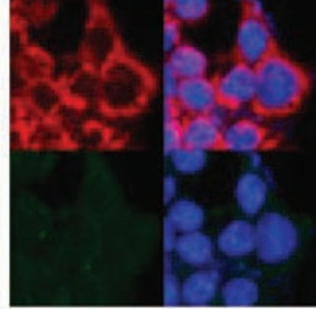

Control+DAPK d/n

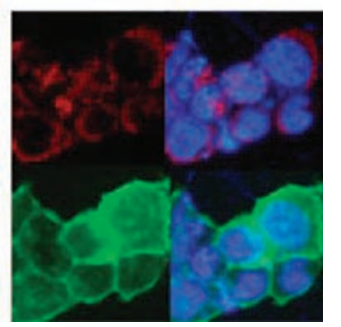

neogenin+DAPK $d / n$

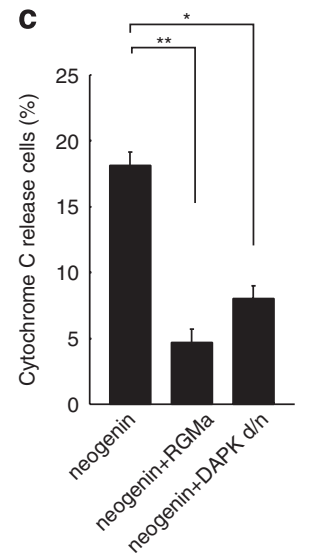

d

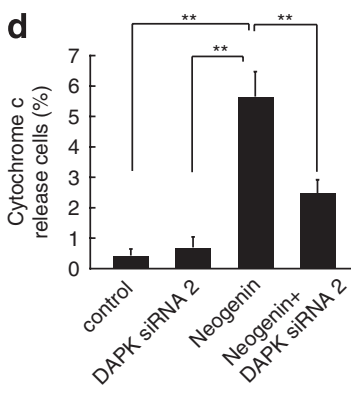

f
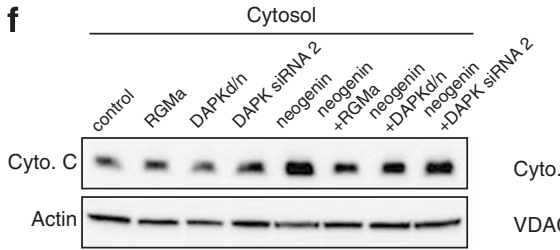

Cyto.
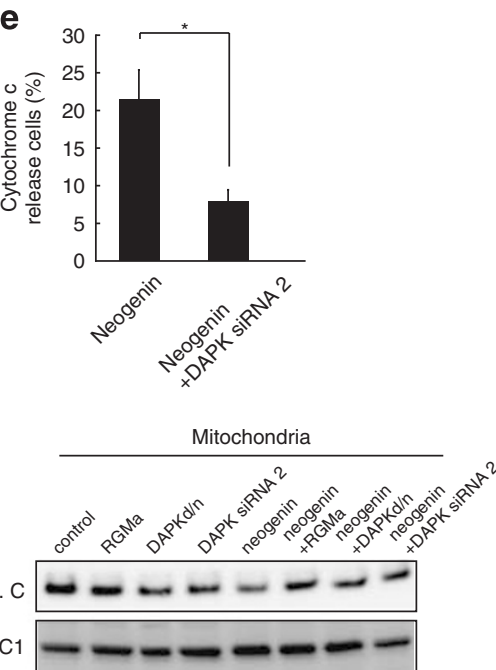

Figure 7 Cytochrome $c$ release from the mitochondria induced by neogenin is mediated by death-associated protein kinase (DAPK). (a) Overexpression of neogenin induced cytochrome $c$ release from the mitochondria in human embryonic kidney (HEK) 293 cells. Cytochrome $c$ release was examined by immunofluorescence with the anti-cytochrome $c$ antibody (red). The cells were immunostained with the anti-VSV-G antibody (for detecting neogenin expression, green) and 4',6'-diamidino-2-phenylindole (DAPI, blue). Note the increase in the number of cells that display diffuse cytochrome $c$ immunoreactivity when the cells were transfected with neogenin (arrows). This neogenin-induced cytochrome $c$ release disappeared on treatment with RGMa or by cotransfection with dominant-negative form of DAPK (DAPK-d/n). Scale bar: $10 \mu \mathrm{m}$. (b, d) Quantification of cells with diffuse cytochrome $c$ staining. The percentage of the number of cytochrome $c$ released cells was quantified. Mean \pm S.E.M. of at least 100 cells from 11 independent experiments is shown. ${ }^{* *} P<0.01$; ${ }^{*} P<0.05$; one-way ANOVA followed by Scheffe's multiple comparison test. (c,e) The ratio of the number of the cells with diffuse cytochrome $c$ staining among the cells expressing VSV-G-tagged neogenin. Mean \pm S.E.M. of at least 100 cells from 11 independent experiments is shown. ${ }^{\star *} P<0.01,{ }^{*} P<0.05$; one-way ANOVA followed by Scheffe's multiple comparison test. (f) Cytosolic and mitochondrial proteins were isolated, and quantified by western blot (upper panels). Quantification of the relative intensity of the band for cytochrome $c$ expression compared with the control (lower graphs). The neogenin overexpression increased the cytosolic cytochrome $c$, whereas transfection of DAPK-d/n or DAPK small-interference RNA (siRNA) as well as the repulsive guidance molecule (RGM) treatment attenuated this effect. ${ }^{* \star} P<0.01$; one-way ANOVA followed by Scheffe's multiple comparison test 
a
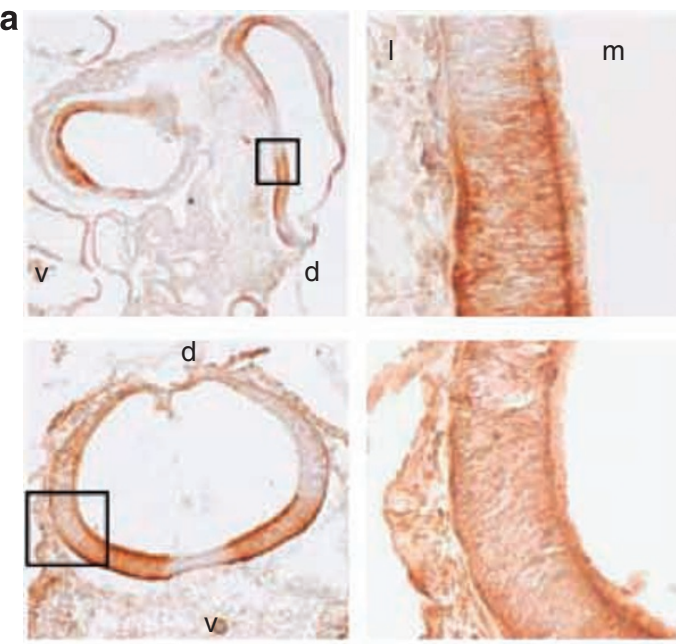

RGM

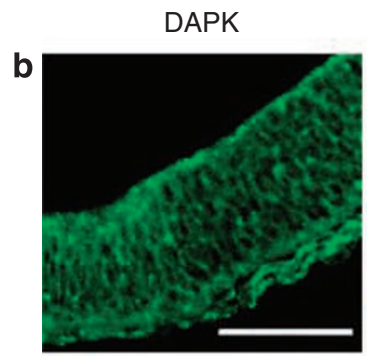

$$
\text { DAPI }
$$
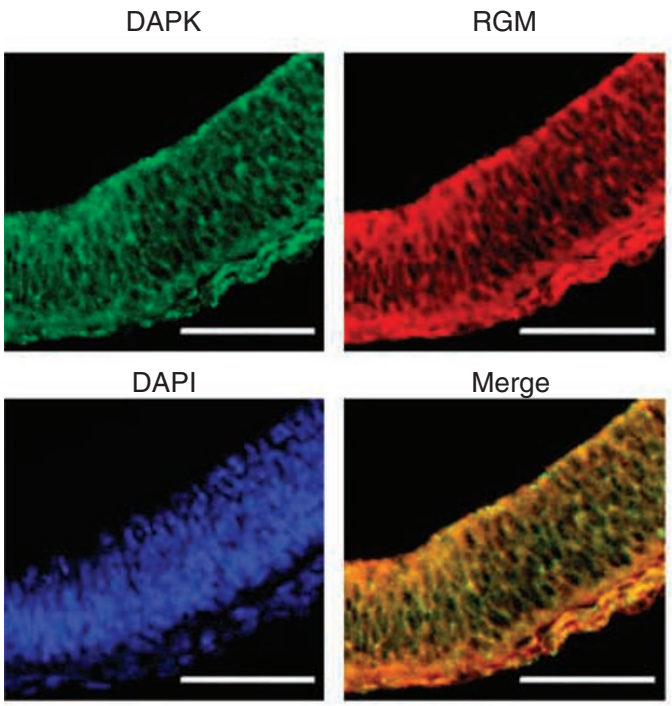

Merge

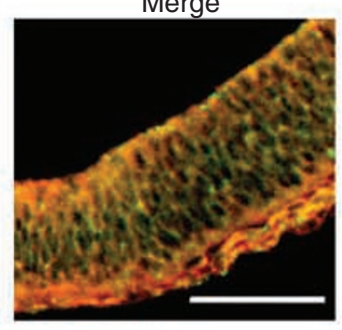

c
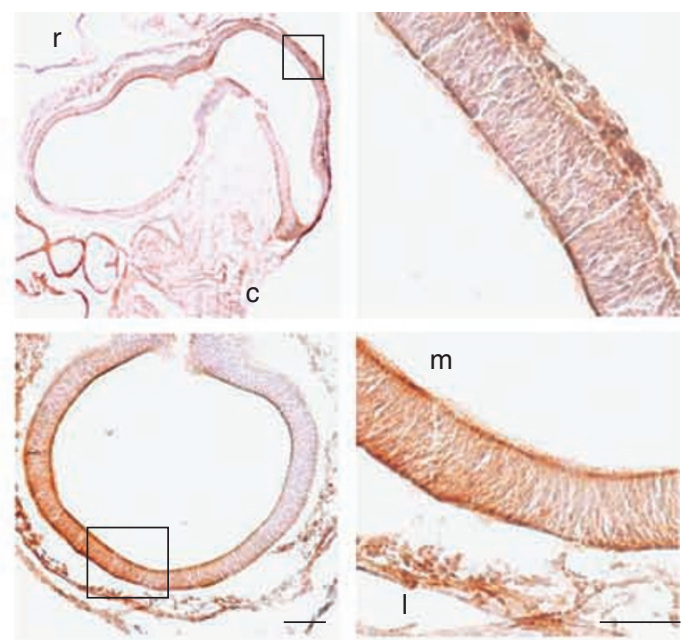

DAPK
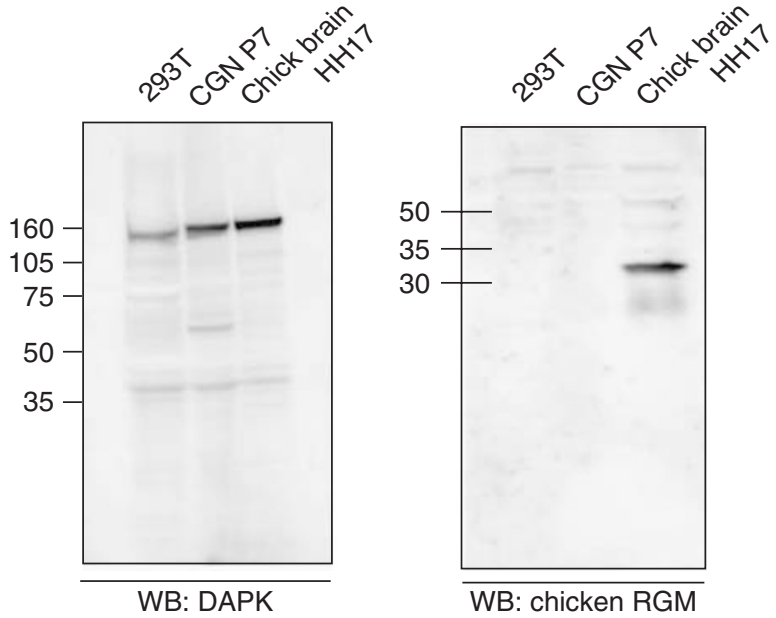

d

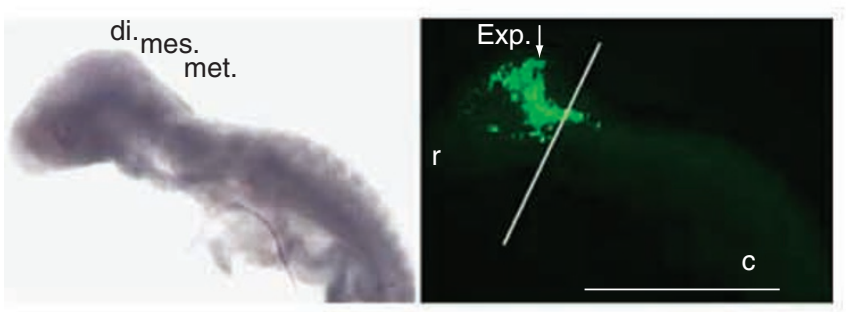

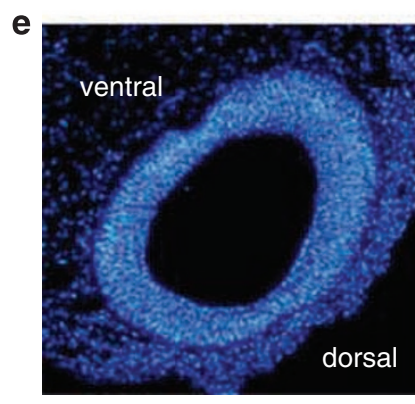

DAPI

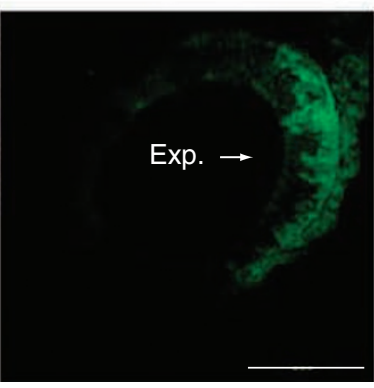

GFP

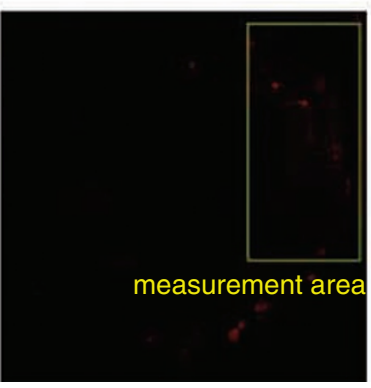

TUNEL 
activity of DAPK is required for neogenin-induced cell death in the developing chick neural tube.

The effects of RGM silencing are mediated by DAPK. RGM expression was repressed using siRNA construct for RGM (RGM siRNA). Electroporation of the RGM siRNA construct into the neural tube indeed repressed the endogenous RGM expression (Figure 10a). Further, immunostaining for GAPDH, DAPK, or neogenin in combination with GFP fluorescence in the neural tube $24 \mathrm{~h}$ after coelectroporation of corresponding siRNA demonstrates that expression of the corresponding protein was repressed in the area where GFP was expressed (Figure 10b). Thus, GFP is a good marker for the siRNA expression. Figure 10c shows the immunostaining for RGM and DAPK-d/n. The signals for GFP (green) and DAPK-d/n (blue) were colocalized in the electroporated side after coelectroporation. Expression of RGM (red) was reduced in the area where GFP (green) as well as DAPK-d/n (blue) was expressed in the neural tube (Figure 10c). The TUNEL staining demonstrated that the electroporation of the RGM siRNA, but not GAPDH siRNA, induced apoptosis on the experimental side (Figures $10 \mathrm{~d}$ and $\mathrm{e}$ ). This confirms that RGM functions as a cell survival factor to counteract neogenin proapoptotic activity. We then examined whether DAPK is associated with the apoptosisinducing effect of RGM silencing. Coelectroporation of the RGM siRNA and DAPK- $d / n$ was performed in the neural tube (Figures $10 \mathrm{~d}$ and e). The number of the TUNEL-positive cells on the experimental side $24 \mathrm{~h}$ after the coelectroporation of RGM siRNA and DAPK-d/n was significantly decreased as compared to that after single electroporation of the RGM siRNA construct (Figures 10d and e). Consistent with this, coelectroporation of DAPK siRNA also reduced the number of the TUNEL-positive cells induced by RGM siRNA (Figures 10d and $e$ ). Coelectroporation of neogenin siRNA reduced the number of the TUNEL-positive cells induced by RGM siRNA (Figures $10 \mathrm{~d}$ and e), supporting the notion that the effect of RGM silencing is mediated by neogenin. These data demonstrate that the cell death is induced in the absence of RGM in the chick neural tube and that the DAPK activity is required for this effect.

\section{Discussion}

To date, various receptors have been identified as dependence receptors, including DCC, p75 neurotrophin receptor, patched, the UNC-5 homologue, integrin receptors $\left(\alpha_{v} \beta_{3}\right.$, $\alpha_{5} \beta_{1}$ ), and the androgen receptor. ${ }^{3} \mathrm{~A}$ comparison of human neogenin and human DCC has revealed that both proteins have identical domain structures and share $50 \%$ amino-acid identity. A high degree of similarity exists between the fourth fibronectin III and the fourth immunoglobulin domain of the extracellular portion. However, the cytoplasmic domains of both proteins are less conserved, with only $37 \%$ identity at the amino-acid level. A considerably higher amino-acid identity $(97 \%)$ is observed in the P3 domain of the ICDs of neogenin and DCC, a region shown to be responsible for focal adhesion kinase (FAK) binding to both proteins. ${ }^{22,23}$ Excluding this small homologous region, the ICD of neogenin shows no similarity with any other protein in the database. Thus, it was suggested that neogenin exerts its proapoptotic activity by using a molecular pathway distinct from DCC or other dependence receptors.

The RGM-neogenin interaction has diverse functions in the developing CNS. In Xenopus embryos, downregulation of RGMa, netrin-1, or neogenin by a morpholino antisense strategy lead to aberrant projections of the supraoptic tract in the forebrain. The aberrant projection phenotype of the RGMa knockdown was very similar in extent and morphology to the neogenin knockdown. ${ }^{7}$ Interestingly, recent report demonstrated that RGM was expressed in the developing retina. ${ }^{24}$ Abnormal topographic targeting of retinotectal projection was found when RGM expression in the retinal ganglion cells was perturbed, suggesting that RGM in the axons is also important in axon guidance. In addition, we observed an unexpected importance of RGMa in early embryonic development when analyzing the RGMa knockout mice. These mice show deficits in neural tube closure at the cephalic level, resulting in an exencephalic phenotype with major morphological defects of the dorsal brain structures. ${ }^{25}$

In the adult CNS, reexpression of RGM is observed following injury and ischemic stroke in the adult human CNS and after a spinal cord injury in rats. ${ }^{26,27}$ Recently, we reported that the inhibition of RGMa enhances the growth of injured axons and promotes functional recovery after spinal cord injury in rats. ${ }^{28}$ Therefore, an interesting hypothesis is that DAPK may kill the neurons after injury or ischemic damage in the CNS but RGM possibly suppresses the DAPK proapoptotic effect as well as axon regeneration. It will be interesting to assess whether DAPK is important in the final cell-fate decision in the pathological state in adults as well as in the developmental stages.

In addition to the importance in inducing cell death, DAPK was recently shown to have apoptosis-independent effects on the cytoskeletons; ${ }^{29}$ it also inhibits integrin activity. ${ }^{16}$ DAPK associates with the stress fibers to induce the inhibition of integrins by the cytoskeletal-binding domain of DAPK. ${ }^{30}$ However, DAPK is not associated with growth cone collapse induced by RGM in our experimental conditions. The association of DAPK with stress fibers hints at its possible

Figure 8 Expression of death-associated protein kinase (DAPK) in the neural tube of chick embryo. (a, b) repulsive guidance molecule (RGM) as well as DAPK is expressed in the neural tube. Cross sections at the level of the mesencephalon from Hamburger Hamilton (HH) stage 17 embryos (20- $\mu \mathrm{m}$ thick sections) were immunostained with their corresponding antibodies. The patchy signals for RGM and DAPK were observed in the neural tube. $v$, ventral; $d$, dorsal; $l$, lateral; $m$, medial; $r$, rostral; $c$, caudal. Scale bar: $200 \mu \mathrm{m}$. (c) Western blot with an anti-RGM or an anti-DAPK antibody. (d) In ovo electroporation. Chick embryos were incubated at $38^{\circ} \mathrm{C}$. The plasmid DNA solution was injected into the $\mathrm{HH}$ stage 10 chick neural tube, and a pulse at $25 \mathrm{~V}$ was applied $5 \times$ for $50 \mathrm{msec}$. AT $24 \mathrm{~h}$ later, the chick embryos were fixed. Green fluorescent protein (GFP) expression (green) was observed in the diencephalons (di), mesencephalon (mes), and metencephalon (met). Scale bar: $1 \mathrm{~mm}$. (e) The signals for terminal deoxynucleotidyl transferase-mediated dUTP nick end labeling (TUNEL, red) and GFP (green) were observed in the electroporated side after coelectroporation with neogenin and GFP constructs. DAPI, DAPI staining. Scale bars: $200 \mu \mathrm{m}$ 
interaction with the RhoA/Rho-kinase pathway. Activation of the RhoA/Rho-kinase pathway is a key signal for axon growth inhibition in the CNS. ${ }^{31}$ Indeed, RGM activates RhoA and Rho-kinase and inhibits neurite growth of the CGNs in vitro by activating RhoA ${ }^{28}$ A possible cross talk between Rho-kinase and DAPK downstream of RGM-neogenin is suggested in that they share the same substrate myosin-regulatory light chain ${ }^{32}$ such interactions should be tested in the future.

\section{Materials and Methods}

Antibodies and plasmid constructs. The primary antibodies used in this study included the monoclonal anti-DAPK antibody (Sigma-Aldrich Inc., St. Louis, a

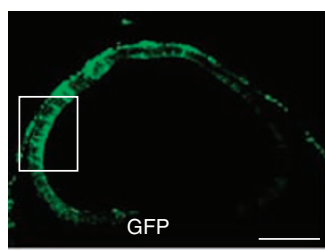

C

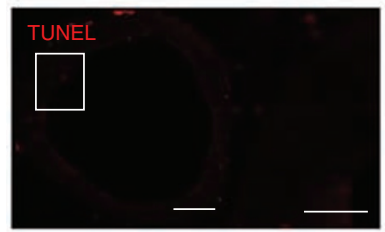

Control

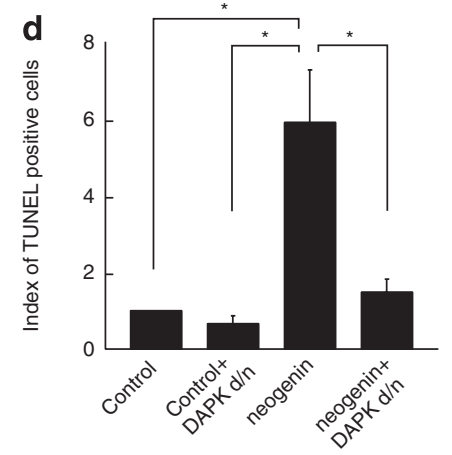

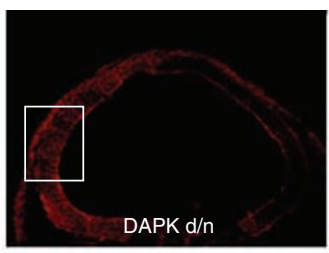

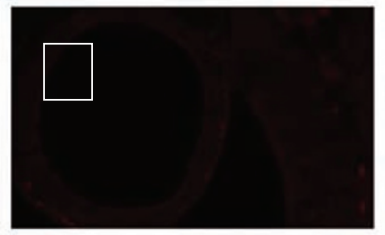

Control+DAPK d/n
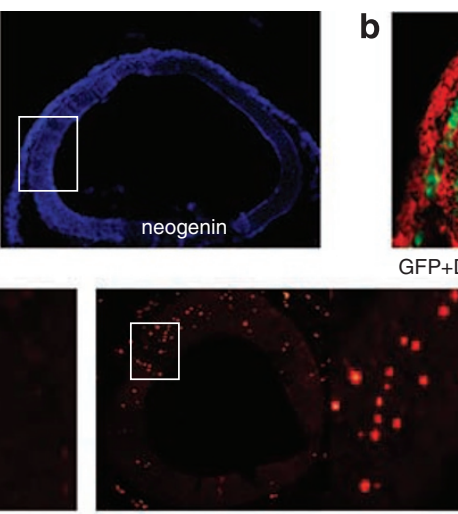

neogenin

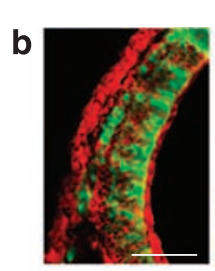

GFP+DAPK $d / n \quad$ GFP+neogenin DAPK $d / n+n e o g e n i n$

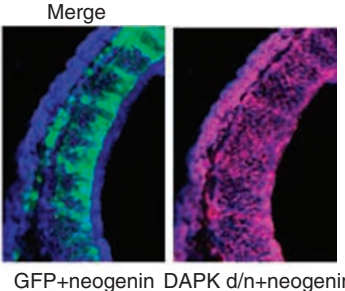

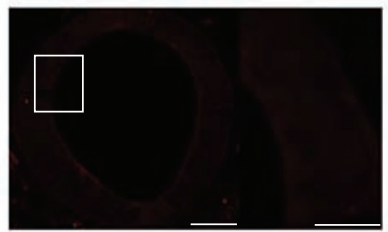

neogenin+DAPK $d / n$
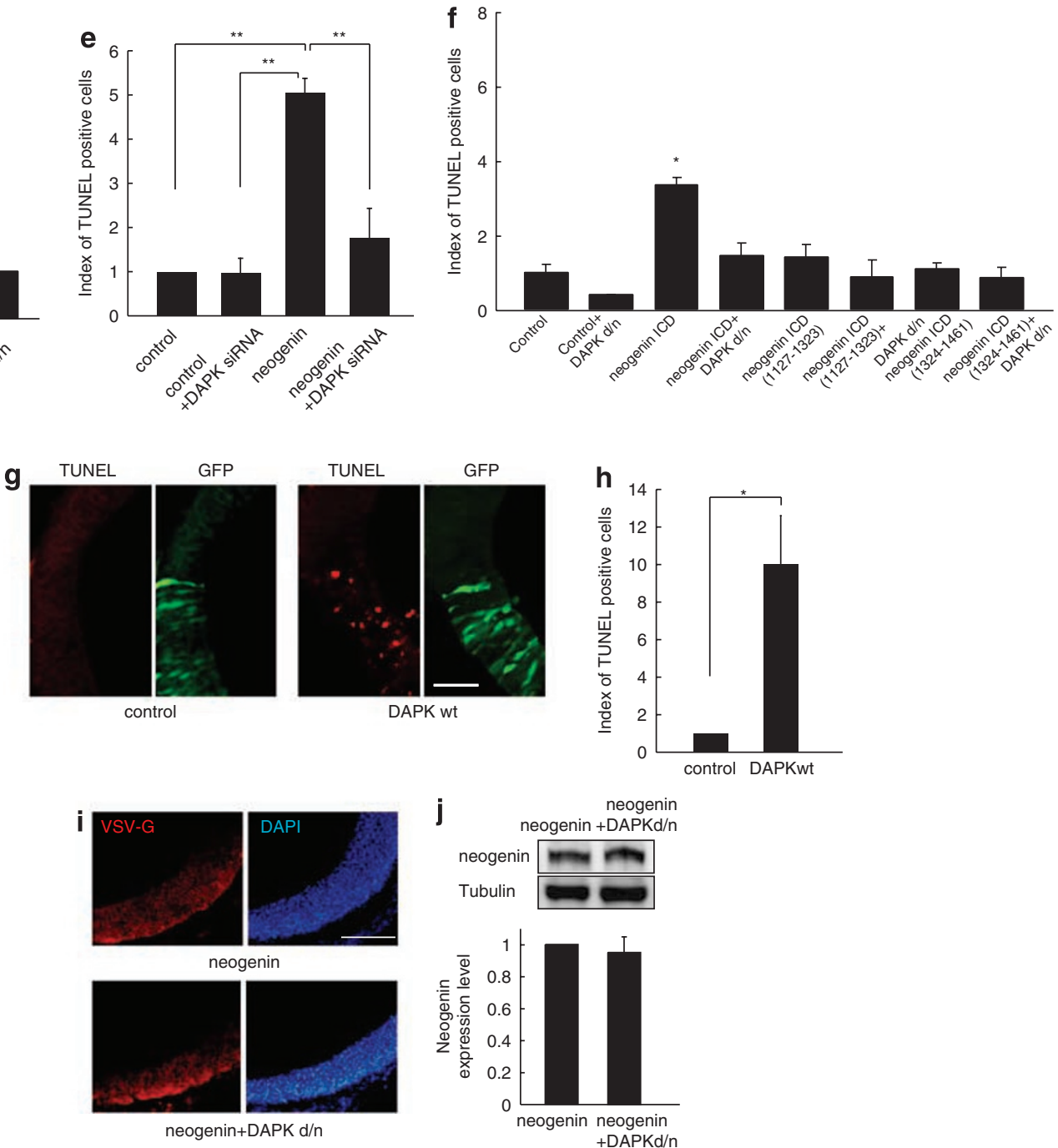
MO, USA), monoclonal anti-phospho-DAPK antibody (pSer308; Sigma-Aldrich Inc.), monoclonal anti-HA antibody (Sigma-Aldrich Inc.), polyclonal anti-VSV-G antibody (Sigma-Aldrich Inc.), polyclonal anti-neogenin antibody (Santa Cruz Biotechnology, CA, USA), polyclonal anti-GST antibody (Santa Cruz Biotechnology), polyclonal anti-His tag antibody (Santa Cruz Biotechnology), polyclonal anti-phospho MLC 2 (Ser19) antibody (Cell Signaling Technology), monoclonal anti-cytochrome $c$ antibody (Promega, Madison, WI, USA; BD Pharmingen), monoclonal anti-GAPDH antibody (Santa Cruz Biotechnology), polyclonal chicken anti-RGM antibody (R\&D Systems Inc., Minneapolis, MN, USA), and polyclonal anti-RGMa antibody, monoclonal against neuronal class III $\beta$-tubulin antibody (Tuj1; Covance Laboratories Inc.), monoclonal anti- $\alpha$-tubulin antibody (Santa Cruz Biotechnology), polyclonal anti- $\beta$-actin antibody (Santa Cruz Biotechnology), and polyclonal anti-VDAC1/Porin antibody (Abcam, Cambridge, UK). The specificity of the polyclonal anti-neogenin antibody against neogenin ${ }^{33}$ and that of the monoclonal anti-phospho-DAPK antibody against phospho-DAPK ${ }^{20}$ was previously reported. The secondary antibodies used include the HRP-linked anti-mouse IgG (Cell Signaling Technology), HRP-linked anti-rabbit IgG (Cell Signaling Technology), Alexa 546-conjugated goat anti-mouse IgG (Molecular Probes, Eugene, OR, USA), Alexa 546-conjugated goat anti-rabbit IgG (Molecular Probes), Alexa 488-conjugated goat anti-rabbit IgG (Molecular Probes), and Alexa 305-conjugated goat anti-rabbit IgG (Molecular Probes). The plasmid constructs encoding various deletions were generated using VSV-G-tagged full-length human neogenin in pcDNA 3.1 (a gift from Dr. Eric Fearon) as a template and the following primers: $5^{\prime}$-CCCAAGCTTGGGCCACCATGTGTACCCGTCGTACCACCT- $3^{\prime}$ and 5'-GCTCTAGAGCTCATTTTCCTAGTCGGTTCATTTCTATGTCTGTGTATGCTGT TGTGATAGCGTTTAGG-3' for the neogenin ICD (neogenin ICD-VSV-G) construct, 5'-CCCAAGCTTGGGCCACCATGTGTACCCGTCGTACCACCT-3' and 5'-GCTCT AGAGCTCATTTTCCTAGTCGGTTCATTTCTATGTCTGTGTAATCAGTGCAGCA TTGTTTGAGACGAA-3' for the C-terminal neogenin ICD (1324-1461) construct, and $5^{\prime}$-GCTAGCGTTTAAACTTAAGCTTGGGCCACCATGCACCAGGACCCTGAA$3^{\prime}$ and $5^{\prime}$-GCTCTAGAGCTCATTTTCCTAGTCGGTTCATTTCTAAGTCTGTGTATG CTGTTGTGATAGCGTTTAGG-3' for the N-terminal neogenin ICD (1127-1323) construct. Rat RGMa siRNA (SmartPool, catalog no. M-082899-00), rat DAPK siRNA (SmartPool, catalog no. L-089981-01), and control siRNA (Non-targetingPool, catalog no. D-001810-10) were purchased from Dharmacon (Lafayette, CO, USA) The sequences of DAPK siRNAs were designed according to Kuo et al..$^{29}$ sense: $5^{\prime}$-CAAGAAACGUUAGCAAAUGUU- $3^{\prime}$ and antisense: $5^{\prime}$-CAUUUGCUAACGU UUCUUGUU-3'; DAPK siRNA 2, sense: $5^{\prime}$-GGUCAAGGAUCCAAAGAAGUU-3' and antisense: $5^{\prime}$-CUUCUUUGGAUCCUUGACCUU-3'. Western blot analysis showed both DAPK siRNA 1 and 2 were effective in reducing the DAPK expression (Figure 5f). For chick RGM siRNA, 5'-CTTCATGCTGGCTGCTTAT-3' was chosen as a target sequence of RGM. For chick neogenin siRNA, CATTACCTCCCACTTCACTF was chosen as a target sequence of neogenin. For chick DAPK siRNA, 5'-GGCGGCGGTCAGCGGTGTA-3' was chosen as a target sequence of DAPK. For a control, the GAPDH siRNA, 5'-GTGGTG GCCATCAATGATC- $3^{\prime}$ was used. To drive siRNA expression in the neural tube, the target $c D N A s$ were inserted in the pSilencer1.0-U6 vector (Ambion, Huntingdon, Cambridgeshire, UK). The wild-type DAPK and the DAPK-d/n in pcDNA3.1 were gifts from Dr. Adi Kimchi.

Cell cultures and transfection. For CGNs, the cerebella of P7-8 rats were removed and mixed with $0.25 \%$ trypsin, triturated, and incubated with $200 \mathrm{U} / \mathrm{ml}$ DNase1 (Takara, Shiga, Japan) for $15 \mathrm{~min}$ at $37^{\circ} \mathrm{C}$. Dulbecco's modified Eagle's medium (DMEM; Invitrogen, Carlsbad, CA, USA) containing $10 \%$ fetal bovine serum (FBS) was added, and the cells were centrifuged at 1000 r.p.m. for 5 min. Neurons were plated on poly-L-lysine-coated chamber slides and were maintained in DMEM/ F12 containing $10 \%$ FBS at $37^{\circ} \mathrm{C}$ in $5 \% \mathrm{CO}_{2}$. CGNs $\left(5 \times 10^{6}\right)$ were suspended in $100 \mu \mathrm{l}$ of Nucleofector solution (Amaxa Biosystems, Cologne, Germany) containing $500 \mathrm{pmol}$ of siRNA. Electroporation was performed by using program 0-003 as described by the manufacturer's protocol (Amaxa Biosystems). Transient transfection of HEK293 cells was performed using Lipofectamine 2000 according to the manufacturer's instructions (Invitrogen). Immunoprecipitation, DAPK phosphorylation assays, and the TUNEL assays were performed $48 \mathrm{~h}$ after transfection.

Coimmunoprecipitation. HEK293 cells were lysed in lysis buffer containing $20 \mathrm{mM}$ Hepes (pH 7.5), $150 \mathrm{mM} \mathrm{NaCl}, 1 \mathrm{mM}$ EDTA, 1\% Triton X-100, 0.1\% SDS and a protease inhibitor mixture (Roche Diagnostics K.K., Tokyo, Japan). The lysates were centrifuged at $20000 \times g$ at $4^{\circ} \mathrm{C}$ for $15 \mathrm{~min}$, and the supernatants were collected. The clear lysates obtained from HEK293 cells were incubated overnight at $4{ }^{\circ} \mathrm{C}$ with the anti-DAPK antibody or the anti-neogenin antibody to pull down endogenous DAPK or neogenin, respectively. The anti-HA antibody or the antiVSV-G antibody was used to pull down HA-tagged DAPK or VSV-G-tagged neogenin, respectively. Rat CGNs were lysed in lysis buffer containing $50 \mathrm{mM}$ Tris$\mathrm{HCl}$ (pH 7.5), $150 \mathrm{mM} \mathrm{NaCl}, 10 \%$ glycerol, 0.5\% Brij-58 (Sigma-Aldrich Inc.), and a protease inhibitor mixture (Roche Diagnostics K.K.). The lysates were centrifuged at $20000 \times g$ at $4^{\circ} \mathrm{C}$ for $10 \mathrm{~min}$, and the supernatants were collected and incubated with either the anti-DAPK antibody or the anti-neogenin antibody to pull down endogenous DAPK or neogenin, respectively. Chick Brains were homogenized in lysis buffer containing $50 \mathrm{mM}$ Tris- $\mathrm{HCl}(\mathrm{pH} 7.5), 150 \mathrm{mM} \mathrm{NaCl}, 1 \mathrm{mM}$ EDTA, $0.5 \%$ NP-40, and a protease inhibitor mixture (Roche Diagnostics K.K.). The lysates were centrifuged at $20000 \times g$ at $4^{\circ} \mathrm{C}$ for $15 \mathrm{~min}$, and the supernatants were collected and incubated with either the anti-DAPK antibody or the anti-neogenin antibody to pull down endogenous DAPK or neogenin, respectively. The immunocomplex was collected using Protein G Sepharose beads (Amersham Biosciences, Piscataway, NJ, USA). The beads were washed $4 \times$ with the lysis buffer. The bound proteins were eluted with $2 \times$ Laemmli sample buffer and subjected to SDS-PAGE followed by immunoblot analysis. The anti-VSV-G antibody or the anti-neogenin antibody was used for detecting neogenin, and the anti-HA antibody or the anti-DAPK antibody was used for DAPK detection. Where indicated, the cells were treated with recombinant mouse RGMa ( $1 \mu \mathrm{g} / \mathrm{ml}$, R\&D Systems) or recombinant mouse netrin-1 $(1 \mu \mathrm{g} / \mathrm{ml}, \mathrm{R} \& \mathrm{D}$ Systems).

Immunodepletion. For immunodepletion, anti-neogenin antibody, or antiDAPK antibody was coupled to Protein G Sepharose beads (Amersham Biosciences), respectively. Rat CGNs were lysed in lysis buffer containing $50 \mathrm{mM}$ Tris- $\mathrm{HCl}$ (pH 7.5), $150 \mathrm{mM} \mathrm{NaCl}, 10 \%$ glycerol, $0.5 \%$ Brij-58 (Sigma-Aldrich Inc.), and a protease inhibitor mixture (Roche Diagnostics K.K.). The lysates were centrifuged at $20000 \times \mathrm{g}$ at $4^{\circ} \mathrm{C}$ for $10 \mathrm{~min}$, and the supernatants were collected and incubated overnight at $4{ }^{\circ} \mathrm{C}$ with the antibody beads. The mixture was centrifuged at $8000 \times g$ at $4{ }^{\circ} \mathrm{C}$ for $10 \mathrm{~min}$ to remove the precipitate and the supernatant was used as immunodepleted conditions in western blot analysis.

Yeast two-hybrid assay. Matchmaker GAL4 Two-Hybrid System 3 (Clontech Laboratories Inc., CA, USA) was used according to the manufacturer's instructions. Various deletion mutants of neogenin ICD and DAPK (see Figure 3)

Figure 9 Cell death induced by neogenin depends on death-associated protein kinase (DAPK) in the neural tube. (a) The signals for green fluorescent protein (GFP, green), dominant-negative form of DAPK (DAPK-d/n, red), and neogenin (blue) were observed in the electroporated side after coelectroporation with neogenin, DAPK-d/n, and GFP constructs. (b) Merged and higher magnification views in (a). (c) Terminal deoxynucleotidyl transferase-mediated dUTP nick end labeling (TUNEL) staining in the neural tube electroporated with the control or the full-length neogenin construct in combination with or without DAPK-d/n. Signals for TUNEL were observed in a lot of cells in the neogenin-electroporated neural tube. Coelectroporation of neogenin in combination with DAPK-d/n resulted in the disappearance of TUNEL-positive cells. A highmagnification view of each photo is shown on the right side. Scale bars: $200 \mu \mathrm{m}$ (low magnification), $100 \mu \mathrm{m}$ (high magnification). (d, e, f, h) Quantification of TUNEL-positive cells. The index factor is the ratio of TUNEL-positive cells on the electroporated side: those in the contralateral side (measurement area, 200-400 $\mu \mathrm{m}$ ). (d, e) Increase in the number of TUNEL-positive cells induced by neogenin intracellular domain (ICD) was abolished by coelectroporation of DAPK-d/n or DAPK small-interference RNA (siRNA). (f) Deletion constructs of neogenin ICD (1127-1323 or 1324-1461) did not increase the number of TUNEL-positive cells. Results are represented as mean \pm S.E.M. $(n=4)$. ${ }^{\star} P<0.05$; ${ }^{\star *} P<0.01$; one-way ANOVA followed by Scheffe's multiple comparison test. (g) Signals for TUNEL were observed in the neural tube after the wild-type DAPK electroporation. (i) Immunohistochemistry for electroporated neogenin. The signals for VSV-G-tagged neogenin (red) were observed in the electroporated side in both groups. Scale bars: $100 \mu \mathrm{m}$. (j) Western blot for electroporated neogenin (upper panel). Quantification of the relative intensity for the neogenin expression (lower graph) 
a

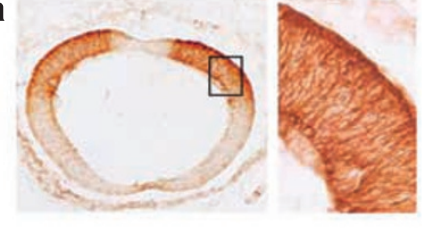

Control

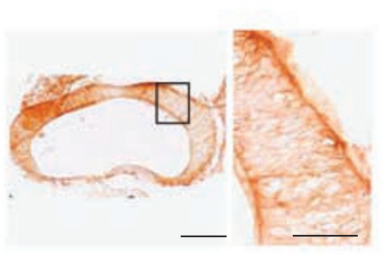

RGM SiRNA

$\underline{\text { b }}$

GAPDH SiRNA

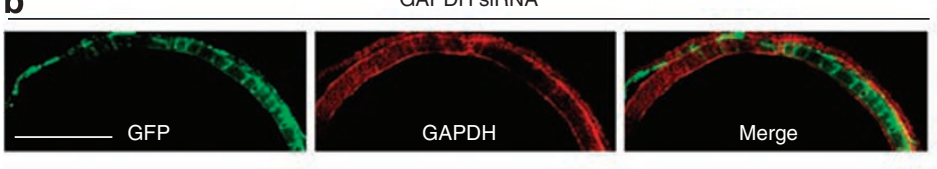

DAPK SIRNA

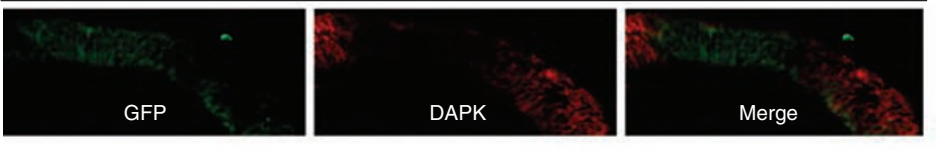

neogenin siRNA

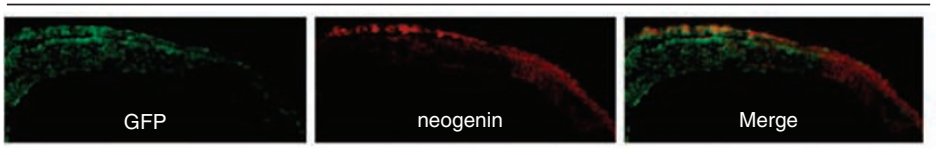

C RGM SiRNA+DAPK $d / n$

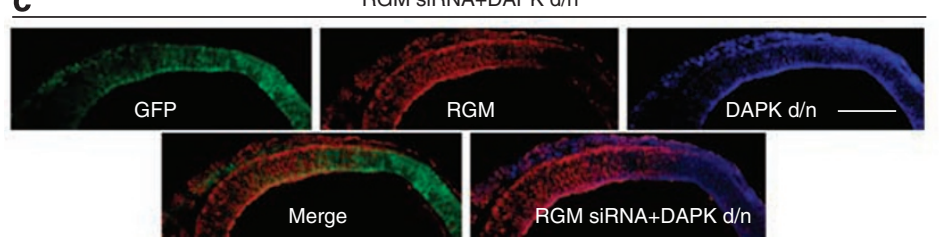

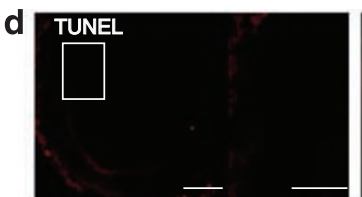

Control

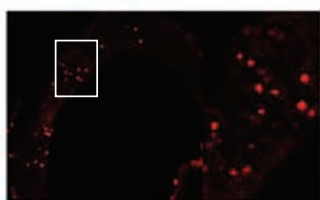

RGM siRNA

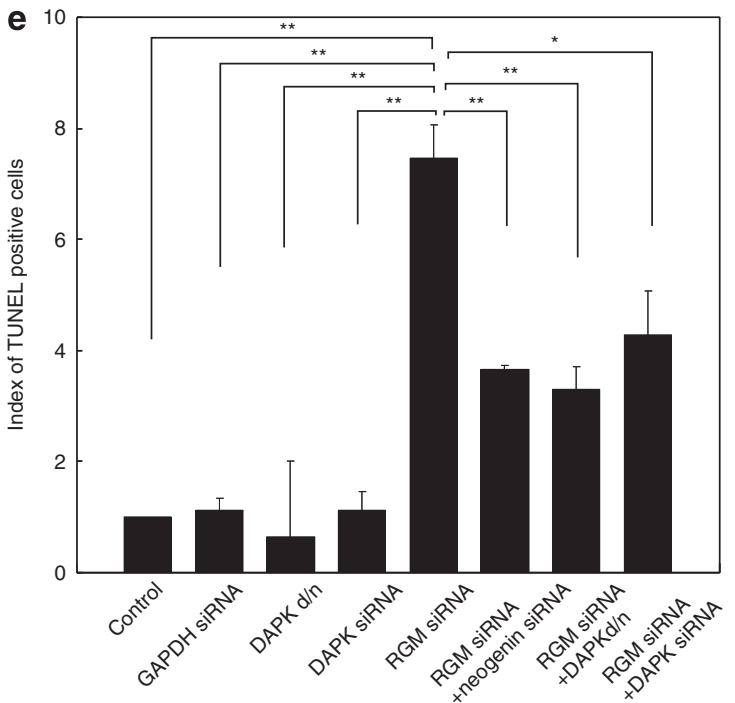

Figure 10 Inhibition of death-associated protein kinase (DAPK) attenuates the cell death induced by repulsive guidance molecule (RGM) knockdown. (a) Immunohistochemistry for RGM. The signals for RGM were observed in the neural tube (control), but were efficiently downregulated in the electroporated side after electroporation with RGM small-interference RNA (siRNA). Scale bars: $200 \mu \mathrm{m}$ (low magnification), $100 \mu \mathrm{m}$ (high magnification). (b) Immunostaining for GAPDH, DAPK, or neogenin in the neural tube $24 \mathrm{~h}$ after coelectroporation of the corresponding siRNA and green fluorescent protein (GFP) constructs. Expression of GAPDH, DAPK, or neogenin on the electroporated side was efficiently reduced. (c) Expression of GFP, RGM, and dominant-negative form of DAPK (DAPK-d/n) after coelectroporation of GFP, RGM siRNA, and DAPK-d/n. Expression of RGM was downregulated in the electroporated side where the signals for GFP (green) and DAPK-d/n (blue) were observed. (d, e) RGM silencing induced cell death in the neural tube, whereas DAPK-d/n or DAPK siRNA repressed it. (d) Terminal deoxynucleotidyl transferase-mediated dUTP nick end labeling (TUNEL) staining in the neural tube electroporated with the RGM siRNA construct in combination with or without DAPK-d/n, DAPK siRNA, and neogenin siRNA constructs. TUNEL staining was performed $24 \mathrm{~h}$ after the electroporation. The number of TUNEL-positive cells was higher on the RGM siRNA-electroporated side only in the absence of DAPK-d/ $n$, DAPK siRNA, and neogenin siRNA constructs. Scale bars: $200 \mu \mathrm{m}$ (low magnification) and $100 \mu \mathrm{m}$ (high magnification). (e) Quantification of the number of TUNEL-positive cells in the neural tube. Results are represented as mean \pm S.E.M. ( $n=4$ ). The index factor is the ratio of TUNEL-positive cells in the electroporated side: those in the contralateral side (measurement area, $200-400 \mu \mathrm{m})$. Results are represented as mean \pm S.E.M. $(n=4)$. ${ }^{* *} P<0.01 ;{ }^{*} P<0.05 ;$ one-way ANOVA followed by Scheffe's multiple comparison test 
were subcloned into the pGBKT7 and pGADT7 vectors, respectively, and the oneon-one transformation assays were performed. The yeast reporter strain AH109 was transformed by the lithium acetate method, and positive clones were selected on plates lacking adenine, histidine, tryptophan, and leucine.

Protein purification and pull-down assay. The DNA fragment encoding the neogenin ICD was cloned to pGEX-6P-3 (GE Healthcare Ltd., UK) vector. His-tagged various fragments of DAPK were cloned to $\mathrm{pET}-29 \mathrm{a}(+)$ vector. Production and purification of the His-tagged proteins were prepared according to the manufacturer's instructions (Novagen). For pull-down assay, equal amounts of GST fusion proteins immobilized on beads were incubated at $4{ }^{\circ} \mathrm{C}$ for $2 \mathrm{~h}$ with Histagged DAPK proteins in GST-binding buffer $(50 \mathrm{mM}$ Hepes $(\mathrm{pH} 7.5), 1 \% \mathrm{NP}-40$ $50 \mathrm{mM} \mathrm{NaCl}, 10 \mathrm{mM}$ EDTA, $20 \%$ glycerol, $1 \mathrm{mM}$ PMSF, $1 \mu \mathrm{g} / \mathrm{ml}$ aprotinin, and $1 \mu \mathrm{g}$ $\mathrm{ml}$ leupeptin). The beads were washed, and proteins bound on beads were analyzed by western blot.

Western blotting. For assessing the phosphorylation state of DAPK, the cells were lysed in lysis buffer containing $50 \mathrm{mM}$ Tris- $\mathrm{HCl}(\mathrm{pH} 7.4), 150 \mathrm{mM} \mathrm{NaCl}, 1 \% \mathrm{NP}$ 40, $2 \mathrm{mM}$ EDTA, $1 \mathrm{mM} \mathrm{NaF}, 1 \mathrm{mM}$ sodium orthovanadate, and the protease inhibito mixture. For chick embryo experiments, the neural tubes from $\mathrm{HH} 17$ embryos were dissected and triturated. They were then incubated in the lysis buffer described above, followed by sonication. These lysates were purified by centrifugation at $20000 \times g$ at $4^{\circ} \mathrm{C}$ for $15 \mathrm{~min}$, and the supernatants were collected and normalized for protein concentration. Equal amounts of proteins were then boiled in sample buffer for $5 \mathrm{~min}$ and were subjected to SDS-PAGE. The proteins were transferred onto a membrane and incubated with primary antibodies. For detection, the ECL chemiluminescence system (GE Healthcare, Piscataway, NJ, USA) and secondary antibodies were used.

Kinase assay. HEK293 cells were lysed in RIPA buffer supplemented with $1 \mathrm{mM}$ PMSF, $10 \mu \mathrm{g} / \mathrm{ml}$ aprotinin, $10 \mu \mathrm{g} / \mathrm{ml}$ aprotinin leupeptin, $1 \mathrm{mM}$ sodium orthovanadate, $4 \mathrm{mM}$ sodium pyrophosphate, and $20 \mathrm{mM} \mathrm{NaF}$. DAPK immunoprecipitated by anti-HA-tagged antibody was incubated with MLC kinase buffer (50 mM Hepes (pH 7.5), $8 \mathrm{mM} \mathrm{MgCl}, 2 \mathrm{mM} \mathrm{MnCl}_{2}, 0.5 \mathrm{mM} \mathrm{CaCl}, 50 \mu \mathrm{M}$ ATP, $0.1 \mathrm{mg} / \mathrm{ml}$ bovine serum albumin (BSA), $1 \mu \mathrm{M} \mathrm{CaM}$ (Sigma-Aldrich Inc.)) at $25^{\circ} \mathrm{C}$ for $10 \mathrm{~min}$. The kinase reactions were resolved by SDS-PAGE, and MLC phosphorylation was detected by immunoblot. The same kinase reactions were subjected to immunoblot analysis to detect the precipitated HA-DAPK. Immunoblot data were quantified by measuring the band intensity (background intensity corresponding to MLC phosphorylation without DAPK was subtracted) using Scion Image software (Scion Corporation, ML, USA).

Immunocytochemistry and immunohistochemistry. Transfected HEK293 cells were fixed for $2 \mathrm{~h}$ or overnight at $4^{\circ} \mathrm{C}$ by using $4 \%$ paraformaldehyde (PFA). Chick embryos were fixed for $30 \mathrm{~min}$ with $4 \%$ PFA $0.2 \%$ glutaraldehyde, and $0.02 \%$ NP-40. For chick tissues, $20-\mu \mathrm{m}$ thick sections were made using a cryostat. The sections or the fixed cells were blocked with PBS containing $10 \% \mathrm{BSA}$ and $0.1 \%$ Triton $\mathrm{X}-100$ for $2 \mathrm{~h}$ at room temperature and were incubated with the appropriate antibodies in PBS containing 5\% BSA Immunoreactivity was visualized using fluorescence-conjugated secondary antibodies or biotinylated secondary antibodies in combination with $\mathrm{ABC}$ system (Vector). Coverslips were then mounted with a mounting medium after DAPI staining for fluorescent staining.

TUNEL and cytochrome $c$ release assay. The TUNEL assay was performed using the ApopTag in situ apoptosis detection kit (Chemicon, Tamecula, CA, USA) according to the manufacturer's instructions. The index factor in the in vitro assays is the relative number of TUNEL-positive cells as compared with the control. The index factor in the in ovo electroporation experiments is the ratio between the number of TUNEL-positive cells on the electroporated side and that on the control side (equal squares were selected for measurement, as indicated in Figure 7d). A minimum of four embryos and three sections per embryo were counted for each experimental condition. For the cytochrome $c$ release assay, the transfected HEK293 cells were immunostained with the anti-cytochrome $c$ antibody. Quantification was performed by measuring the number of the cells with diffuse cytochrome $c$ staining patterns among all the cells or the cells expressing VSV-G neogenin. For cell fractionation analysis, cytosolic and mitochondrial proteins were isolated using a commercially available mitochondria/cytosol fractionation kit (BioVision). Cells were collected by centrifugation at $600 \times g$ for $5 \mathrm{~min}$ and resuspended in kit-provided cytosol extraction buffer containing protease inhibitor mixture and dithiothreitol. After incubation on ice for $10 \mathrm{~min}$, the cells were homogenized on ice. Homogenizations were centrifuged at $700 \times g$ for $10 \mathrm{~min}$ at $4^{\circ} \mathrm{C}$, and the supernatants were collected. Then, the collected supernatants were centrifuged again at $10000 \times \mathrm{g}$ for $30 \mathrm{~min}$ at $4^{\circ} \mathrm{C}$. The resulting supernatants were designated as cytosolic fractions, and the pellets were resuspended in kit-provided mitochondria extraction buffer and designated as mitochondrial fractions. Protein content was determined using BCA protein assay kit (Pierce, Rockford, IL, USA). Equivalent amounts of mitochondrial and cytosolic proteins were separated on SDS-PAGE and transferred onto membranes. Then, western blot analysis was performed. $\beta$-actin (cytosol) and VDAC1 (mitochondria) served as loading normalization

In ovo electroporation. Fertilized chicken eggs were obtained from a local farm and were incubated at $38^{\circ} \mathrm{C}$. Various constructs in the solution were injected into $\mathrm{HH}$ stage 10 chick embryos by in ovo electroporation. These included human VSV-G-tagged full-length neogenin in pcDNA3.1 $(1.0 \mathrm{mg} / \mathrm{ml})$, siRNA plasmids $(1.0 \mathrm{mg} / \mathrm{ml})$, DAPK wild type in pcDNA3.1 $(1.0 \mathrm{mg} / \mathrm{ml})$, and DAPK-d $/ \mathrm{n}$ in pcDNA3.1 $(1.3 \mathrm{mg} / \mathrm{ml})$. Electroporation was performed using a CUY21 electroporator and a CUY-610 standard electrode (NEPA gene, Chiba, Japan). We carried out electroporation by using five pulses of $50-\mathrm{ms}$ duration at $25 \mathrm{~V}$. The GFPexpression vector (pcDNA3.1, $0.5 \mathrm{mg} / \mathrm{ml}$ ) was coelectroporated to assess the electroporation efficiency. At $24 \mathrm{~h}$ later, the embryos were fixed and then subjected to the TUNEL assay and immunohistochemistry.

Growth cone collapse assay. CGNs were transfected with control siRNA or DAPK siRNA by using nucleofection. After $48 \mathrm{~h}$ culture, cells were treated with or without soluble RGMa $(2 \mu \mathrm{g} / \mathrm{ml})$ for $30 \mathrm{~min}$. Then, the cells were fixed with $0.5 \%$ glutaraldehyde for $1 \mathrm{~h}$ at room temperature, and treated with PBS containing $5 \%$ BSA and $0.1 \%$ Triton $\mathrm{X}-100$. The cells were incubated with anti-Tuj1 antibody and rhodamine-phalloidin for $2 \mathrm{~h}$ at room temperature, followed by incubation with antimouse IgG antibody conjugated with Alexa Fluor 568 (Invitrogen) for $1 \mathrm{~h}$ at room temperature. Finally, they were analyzed using Olympus BX51 microscopy.

Acknowledgements. This work was supported by a Research Grant from the National Institute of Biomedical Innovation (05-12) and Grant-in-Aid for Young Scientists (S) from JSPS. We thank Tetsuichiro Saito and Yuko Muroyama for technical guidance for in ovo electroporation

1. Stahl B, Muller B, von Boxberg Y, Cox EC, Bonhoeffer F. Biochemical characterization of a putative axonal guidance molecule of the chick visual system. Neuron 1990; $\mathbf{5}$ : 735-743.

2. Monnier PP, Sierra A, Macchi P, Deitinghoff L, Andersen JS, Mann M et al. RGM is a repulsive guidance molecule for retinal axons. Nature 2002; 419: 392-395.

3. Matsunaga $E$, Chedotal A. Repulsive guidance molecule/neogenin: a novel ligand-receptor system playing multiple roles in neural development. Dev Growth Differ 2004; 46: 481-486.

4. Mueller BK, Yamashita T, Schaffar G, Mueller R. The role of repulsive guidance molecules in the embryonic and adult vertebrate central nervous system. Philos Trans $R$ Soc Lond $B$ Biol Sci 2006; 361: 1513-1529.

5. Rajagopalan S, Deitinghoff L, Davis D, Conrad S, Skutella T, Chedotal A et al. Neogenin mediates the action of repulsive guidance molecule. Nat Cell Biol 2004; 6 : 756-762.

6. Vielmetter J, Kayyem JF, Roman JM, Dreyer WJ. Neogenin, an avian cell surface protein expressed during terminal neuronal differentiation, is closely related to the human tumor suppressor molecule deleted in colorectal cancer. J Cell Biol 1994; 127: 2009-2020.

7. Wilson $\mathrm{NH}$, Key B. Neogenin interacts with RGMa and netrin-1 to guide axons within the embryonic vertebrate forebrain. Dev Biol 2006; 296: 485-498.

8. Matsunaga $\mathrm{E}$, Nakamura $\mathrm{H}$, Chedotal $\mathrm{A}$. Repulsive guidance molecule plays multiple roles in neuronal differentiation and axon guidance. J Neurosci 2006; 26: 6082-6088.

9. Fitzgerald DP, Cole SJ, Hammond A, Seaman C, Cooper HM. Characterization of neogenin-expressing neural progenitor populations and migrating neuroblasts in the embryonic mouse forebrain. Neuroscience 2006; 142: 703-716.

10. Matsunaga E, Tauszig-Delamasure S, Monnier PP, Mueller BK, Strittmatter SM, Mehlen P et al. RGM and its receptor neogenin regulate neuronal survival. Nat Cell Biol 2004; 6: 749-755.

11. Bredesen DE, Mehlen $P$, Rabizadeh S. Apoptosis and dependence receptors: a molecular basis for cellular addiction. Physiol Rev 2004; 84: 411-430.

12. Mehlen $P$, Fearon ER. Role of the dependence receptor DCC in colorectal cancer pathogenesis. J Clin Oncol 2004; 22: 3420-3428.

13. Deiss LP, Feinstein E, Berissi H, Cohen O, Kimchi A. Identification of a novel serine/ threonine kinase and a novel $15-\mathrm{kD}$ protein as potential mediators of the gamma interferoninduced cell death. Genes Dev 1995; 9: 15-30. 
14. Cohen O, Inbal B, Kissil JL, Raveh T, Berissi H, Spivak-Kroizaman T et al. DAP-kinase participates in TNF-alpha- and Fas-induced apoptosis and its function requires the death domain. J Cell Biol 1999; 146: 141-148.

15. Jang $\mathrm{CW}$, Chen $\mathrm{CH}$, Chen $\mathrm{CC}$, Chen JY, Su YH, Chen RH. TGF-beta induces apoptosis through Smad-mediated expression of DAP-kinase. Nat Cell Biol 2002; 4: 51-58.

16. Wang WJ, Kuo JC, Yao CC, Chen RH. DAP-kinase induces apoptosis by suppressing integrin activity and disrupting matrix survival signals. J Cell Biol 2002; 159: 169-179.

17. Cohen O, Feinstein E, Kimchi A. DAP-kinase is a Ca2+/calmodulin-dependent cytoskeletal-associated protein kinase, with cell death-inducing functions that depend on its catalytic activity. EMBO J 1997; 16: 998-1008.

18. Zhu XJ, Wang CZ, Dai PG, Xie $Y$, Song NN, Liu $Y$ et al. Myosin $X$ regulates netrin receptors and functions in axonal path-finding. Nat Cell Biol 2007; 9: 184-192.

19. Shohat G, Spivak-Kroizman T, Cohen O, Bialik S, Shani G, Berrisi H et al. The proapoptotic function of death-associated protein kinase is controlled by a unique inhibitory autophosphorylation-based mechanism. J Biol Chem 2001; 276: 47460-47467.

20. Llambi F, Lourenco FC, Gozuacik D, Guix C, Pays L, Del Rio G et al. The dependence receptor UNC5H2 mediates apoptosis through DAP-kinase. EMBO J 2005; 24: 1192-1201.

21. Green DR, Reed JC. Mitochondria and apoptosis. Science 1998; 281: 1309-1312.

22. Ren XR, Ming GL, Xie Y, Hong Y, Sun DM, Zhao ZQ et al. Focal adhesion kinase in netrin1 signaling. Nat Neurosci 2004; 7: 1204-1212.

23. Williams ME, Strickland $P$, Watanabe $K$, Hinck L. UNC5H1 induces apoptosis via its juxtamembrane region through an interaction with NRAGE. J Biol Chem 2003; 278 17483-17490.

24. Tassew NG, Chestopolava L, Beecroft R, Matsunaga E, Teng H, Chedotal A et al. Intraretinal RGMa is involved in retino-tectal mapping. Mol Cell Neurosci 2008; 37 761-769.
25. Niederkofler V, Salie R, Sigrist M, Arber S. Repulsive guidance molecule (RGM) gene function is required for neural tube closure but not retinal topography in the mouse visual system. J Neurosci 2004; 24: 808-818.

26. Schwab JM, Conrad S, Monnier PP, Julien S, Mueller BK, Schluesener HJ. Spinal cord injury-induced lesional expression of the repulsive guidance molecule (RGM). Eur $J$ Neurosci 2005; 21: 1569-1576.

27. Schwab JM, Monnier PP, Schluesener HJ, Conrad S, Beschorner R, Chen L et al. Central nervous system injury-induced repulsive guidance molecule expression in the adult human brain. Arch Neurol 2005; 62: 1561-1568.

28. Hata K, Fujitani M, Yasuda Y, Doya H, Saito T, Yamagishi $S$ et al. RGMa inhibition promotes axonal growth and recovery after spinal cord injury. J Cell Biol 2006; 173: $47-58$.

29. Kuo JC, Wang WJ, Yao CC, Wu PR, Chen RH. The tumor suppressor DAPK inhibits cell motility by blocking the integrin-mediated polarity pathway. J Cell Biol 2006; 172: 619-631.

30. Bialik S, Bresnick AR, Kimchi A. DAP-kinase-mediated morphological changes are localization dependent and involve myosin-II phosphorylation. Cell Death Differ 2004; 11 : 631-644.

31. Mueller BK, Mack H, Teusch N. Rho kinase, a promising drug target for neurological disorders. Nat Rev Drug Discov 2005; 4: 387-398.

32. Velentza AV, Schumacher AM, Watterson DM. Structure, activity, regulation, and inhibitor discovery for a protein kinase associated with apoptosis and neuronal death. Pharmaco Ther 2002; 93: 217-224.

33. Zhang AS, West Jr AP, Wyman AE, Bjorkman PJ, Enns CA. Interaction of hemojuvelin with neogenin results in iron accumulation in human embryonic kidney 293 cells. J Biol Chem 2005; 280: 33885-33894.

\section{Supplementary Information accompanies the paper on Cell Death and Differentiation website (http://www.nature.com/cdd)}

